\title{
Pseudo-Casimir stresses and elasticity of a confined elastomer film
}

\author{
Bing-Sui $\mathrm{Lu}^{1}{ }^{\text {* }}$ Ali Naji ${ }^{2}$, and Rudolf Podgornik ${ }^{1}$ \\ ${ }^{1}$ Department of Theoretical Physics, J. Stefan Institute, 1000 Ljubljana, Slovenia. \\ ${ }^{2}$ School of Physics, Institute for Research in Fundamental Sciences (IPM), P.O. Box 19395-5531, Tehran, Iran
}

(Dated: August 6, 2021)

\begin{abstract}
Investigations of the elastic behavior of bulk elastomers have traditionally proceeded on the basis of classical rubber elasticity, which regards chains as thermally fluctuating but disregards the thermal fluctuations of the cross-links. Here, we consider an incompressible and flat elastomer film of an axisymmetric shape confined between two large hard co-planar substrates, with the axis of the film perpendicular to the substrates. We address the impact that thermal fluctuations of the crosslinks have on the free energy of elastic deformation of the system, subject to the requirement that the fluctuating elastomer cannot detach from the substrates. We examine the behavior of the deformation free energy for one case where a rigid pinning boundary condition is applied to a class of elastic fluctuations at the confining surfaces, and another case where the same elastic fluctuations are subjected to soft "gluing" potentials. We find that there can be significant departures (both quantitative and qualitative) from the prediction of classical rubber elasticity theory when elastic fluctuations are included. Finally, we compare the character of the attractive part of the elastic fluctuation-induced, or pseudo-Casimir, stress with the standard thermal Casimir stress in confined but non-elastomeric systems, finding the same power law decay behavior when a rigid pinning boundary condition is applied; for the case of the gluing potential, we find that the leading order correction to the attractive part of the fluctuation stress decays inversely with the inter-substrate separation.
\end{abstract}

PACS numbers: 81.05.Lg, 61.41.+e, 05.20.-y

\section{INTRODUCTION}

There has been increasing interest in polymer network films of micron to nanoscale thicknesses [1], which can be used as sensors that mimic biological organs, tunable Bragg reflectors and synthetic cell substrata [2, 3]. Natural polymer network films also exist, for example, the actin filament networks of cells and the intertwining sphingomyelin chains in a myelin sheath [4]. A conventional picture of a polymer network is that of a collection of chains whose end-points (or junctures) are permanently cross-linked into an elastic matrix that undergoes deformation [5]. The shear modulus emerges from the entropic cost of thermal fluctuations of the chains [6 9]. On the other hand, the elastic matrix itself is also a thermally fluctuating entity at room temperature, with the chain end-points undergoing thermally driven displacements about their mean positions [7, 10 12]. The end-points have a typical localization length or root-mean-square displacement that is directly related to how densely the network is cross-linked [10]. Physically the typical localization length reflects how strongly localized the network constituents are, i.e., a smaller value would correspond to a stronger localization. We can also regard it as a cut-off length scale below which continuum elasticity theory no longer applies, and it is in this sense that we shall apply the term in the rest of the Paper. Thus quantities such as strain and deformation tensor are really coarse-grained quantities that have a mean-

*Electronic address: binghermes@gmail.com ing only on length scales larger than the typical localization length. The classical theory of rubber elasticity [6] (also known as the affine network model [8]) takes into account the thermal fluctuations of the chains, but regards the elastic matrix (i.e., the cross-linked ends of the fluctuating chains) as thermally non-fluctuating. However, the effects of thermal fluctuations of the elastic matrix (henceforth called "elastic fluctuations") can substantially modify the predictions of classical rubber elasticity when the local incompressibility of the elastomer is taken into account, as has been shown for the case of a bulk elastomer (i.e., whose dimensions are all much larger than the typical localization length-scale) [11]. It is thus of interest to study how elastic fluctuations can modify the elastic deformation behavior of a thin locally incompressible elastomer film confined between two large hard co-planar substrates. This would involve the interplay between elastic fluctuations and the finite-size effects of the elastomer system. Here and in what follows, we take "thin" to refer to a thickness that is much smaller than the square root of the cross-sectional area of the elastomer surface co-planar with the substrates, but still larger than the typical localization length.

Our paper represents a first step in the theoretical investigation of the effects of thermal elastic fluctuations on the behavior of a confined incompressible elastomer film. In this confined system boundary conditions have to be imposed on the elastic fluctuations at the confining surfaces. We address the case of a boundary condition (BC) that enforces the vanishing of elastic fluctuations at the confining surfaces (i.e., the "rigid pinning" BC, which is mathematically equivalent to a Dirichlet BC), as well as the case where we have a "soft" gluing po- 
tential (instead of a "hard" Dirichlet BC) acting at each confining surface on a class of elastic fluctuations. From studies in other systems, confinement has been known to result in fluctuation-induced, Casimir or pseudo-Casimir stresses 13 18]. Such stresses are the result of thermal or quantum fluctuations of a field with long range correlation, constrained by the presence of boundary surfaces. The surfaces "feel" the presence of each other via these fluctuating fields. The long range correlations can emerge for example in ordered soft matter systems which exhibit broken symmetry (i.e., an ordered phase), where the corresponding Goldstone modes mediate the pseudoCasimir force between the boundary surfaces of the system. In fluctuating elastomers, there are elastic phonons which are the coarse-grainings of thermally excited random displacements of the cross-linking points. As we will see later, these phonons are "massless" (i.e., they only appear as gradient terms in the Hamiltonian), and it is well-known that the phonons can therefore exhibit long-range correlations [11]. Thus, we expect analogous pseudo-Casimir stresses to arise in a thermally fluctuating confined elastomer. In confined nematics 15, 16 and confined polymer liquid crystals [17], the fluctuationinduced pseudo-Casimir stress is predicted to be attractive and decay as the third power of inverse inter-surface separation. The route is thus open to the following interrelated pair of investigations. Firstly, from the angle of rubber elasticity, how do thermal elastic fluctuations modify the elastic behavior predicted by classical rubber theory, for a confined elastomer film? In particular, how does the type of boundary conditions impact on the deformation free energy behavior? Secondly, from the purview of the field of fluctuation-induced forces [18], how does the character of the attractive part of the elastic fluctuation-induced stress in a confined elastomer compare with the Casimir stress induced by confined electromagnetic, nematic, or polymer liquid crystal fluctuation modes?

In the interest of simplicity, we disregard the effects of disorder introduced by the heterogeneous distribution of cross-links, and we focus instead on homogeneous elastomers, in which every point of the elastomer (together with its thermal fluctuations) undergoes an affine transformation under an externally applied uniaxial shear deformation. We study the case of incompressible elastomers as the shear modulus of rubber is substantially smaller than its bulk modulus (often by at least two orders of magnitude [9]), which justifies the approximation of incompressibility. We also limit our consideration to elastomer films whose thicknesses are larger than the typical localization lengthscale (and thus of macroscopic lengthscales), in order that the framework of continuum elasticity theory may still be used, but the thickness is much smaller than the transverse dimension of the film, so that the finite-size effects on elastic fluctuations cannot be neglected.

\section{THE HAMILTONIAN}

Our system is an elastomer film which in its undeformed state has a certain thickness $L$ and cross-sectional area $S$, and is confined between a pair of co-planar hard substrates. The elastomer film is then subjected to a uniaxial shear deformation. To describe the corresponding energy cost for a given thermal configuration of the elastomer, we take the Hamiltonian of Ref. [11]:

$$
H_{e l}=\frac{\mu_{0}}{2} \int_{0}^{L} d z \int d^{2} x_{\perp} \frac{\partial R_{i}(\mathbf{x})}{\partial x_{a}} \frac{\partial R_{i}(\mathbf{x})}{\partial x_{a}}
$$

where $\mathbf{R}(\mathbf{x})=\underline{\underline{\Lambda}} \cdot(\mathbf{x}+\mathbf{u}(\mathbf{x}))$. Here $\underline{\underline{\Lambda}}$ is the deformation gradient, $\mathbf{x}$ is the mean position of a given mass-point in the elastomer prior to deformation, and $\mathbf{u}(\mathbf{x})$ denotes the elastic phonon, i.e., the thermal fluctuation of the masspoint about its mean position. The coordinate $\mathbf{x}$ can equally well be regarded as a label affixed to each masspoint in the undeformed elastomer, which tags along as the mass-point moves when the elastomer is deformed.

\section{PARTITION FUNCTION}

The partition function is given by

$$
Z=\int \mathcal{D} \mathbf{u} \delta(\nabla \cdot \mathbf{u}) e^{-\beta H_{e l}} \equiv Z_{0} Z_{\mathbf{u}}
$$

Here we have decomposed $Z$ into a fluctuation-free, mean-field part $Z_{0}$, and a fluctuation contribution $Z_{\mathbf{u}}$. The symbol $\delta(f)$ denotes the Dirac delta-functional, which is defined to be zero (non-zero) if $f \neq 0(f=$ 0) [19]. By writing the Dirac delta-function inside the functional integral over $\mathbf{u}$, we are enforcing the local incompressibility of the elastomer: $\nabla \cdot \mathbf{u}=0$. This linear constraint is true only for small $\mathbf{u}$, which is the regime we consider. This constraint is derived from the more general nonlinear local incompressibility constraint, viz., $\operatorname{det} \partial \mathbf{R} / \partial \mathbf{x}=1$. We can see this by writing $\operatorname{det} \partial \mathbf{R} / \partial \mathbf{x}=$ $\operatorname{det}(\underline{\Lambda}) \operatorname{det}(\underline{\delta}+\partial \mathbf{u} / \partial \mathbf{x})$, and using the global incompressibility of the elastomer, viz., $\operatorname{det}(\underline{\underline{\Lambda}})=1$, which forces $0=\ln \operatorname{det}(\underline{\underline{\delta}}+\partial \mathbf{u} / \partial \mathbf{x})=\operatorname{Tr} \ln (\underline{\underline{\delta}}+\overline{\bar{\partial}} \mathbf{u} / \partial \mathbf{x})$. For small $\mathbf{u}$ we can expand the logarithm to linear order, and obtain $\nabla \cdot \mathbf{u}=0$.

The mean-field free energy is given by

$$
F_{0}=-k_{\mathrm{B}} T \ln Z_{0}=\frac{\mu_{0}}{2} V \operatorname{Tr}\left(\underline{\underline{\Lambda}}^{\mathrm{T}} \cdot \underline{\underline{\Lambda}}\right) .
$$

Taking the $z$-direction to be perpendicular to the crosssectional surface of the elastomer, a uniaxial shear deformation is described by the deformation gradient: $\underline{\underline{\Lambda}}=$ $\operatorname{diag}\left(\lambda^{-1 / 2}, \lambda^{-1 / 2}, \lambda\right)$, where $\lambda>1(\lambda<1)$ corresponds to uniaxial extension (compression). Correspondingly, the mean-field free energy becomes

$$
F_{0}=\frac{\mu_{0}}{2} V\left(\lambda^{2}+\frac{2}{\lambda}\right) \text {. }
$$


The elastic fluctuation correction to $Z$ is described by

$$
Z_{\mathbf{u}} \equiv \int \mathcal{D} \mathbf{u} \delta(\nabla \cdot \mathbf{u}) e^{-\beta H_{\mathbf{u}}}
$$

where $H_{\mathbf{u}}$ is the Hamiltonian for elastic fluctuations. As we show in Appendix $\mathrm{A}, H_{\mathbf{u}}$ is given by

$$
H_{\mathbf{u}}=\frac{\mu_{0}}{2} \int d^{3} x \partial_{a} u_{b} \Lambda_{b i}^{\mathrm{T}} \Lambda_{i c} \partial_{a} u_{c} .
$$

We can thus also express $H_{e l}$ as

$$
H_{e l}=\frac{\mu_{0}}{2} \int_{0}^{L} d z \int d^{2} x_{\perp}\left(\Lambda_{a i}^{\mathrm{T}} \Lambda_{i a}+\partial_{a} u_{b} \Lambda_{b i}^{\mathrm{T}} \Lambda_{i c} \partial_{a} u_{c}\right) .
$$

The first term describes classical rubber elasticity, and is derived by considering the entropy of fluctuating Gaussian chains with end-points fixed in a thermally nonfluctuating matrix that deforms affinely. On the other hand, the second term allows for the thermal fluctuations of the end-points themselves.

\section{A. Boundary conditions}

Furthermore, we need to specify boundary conditions (BC) for the elastic fluctuations $\boldsymbol{u}$ at the two interfaces. Owing to the local incompressibility constraint, the BC can only be enforced on two components of $\boldsymbol{u}$. Let us write $\boldsymbol{u}=\left(\mathbf{v}, u_{z}\right)$ and make a Helmholtz decomposition of $\mathbf{v}$ into an irrotational and a solenoidal part: $\mathbf{v}=\mathbf{v}^{\|}+$ $\mathbf{v}^{\perp}$. The solenoidal fluctuation, $\mathbf{v}^{\perp}$, satisfies $\nabla_{\perp} \cdot \mathbf{v}^{\perp}=$ 0 , whilst the irrotational fluctuation, $\mathbf{v}^{\|}$, satisfies $\nabla_{\perp} \times$ $\mathbf{v}^{\|}=0$. The symbol $\nabla_{\perp} \equiv\left(\partial_{x}, \partial_{y}\right)$ refers to the twodimensional gradient operator. The first set of boundary conditions are the non-detachment $\mathrm{BC}$ :

$$
u_{z}(z=0)=u_{z}(z=L)=0,
$$

which enforce the condition that the surfaces of the elastomer do not detach from the substrates [20]. Regarding the second set of boundary conditions for the other components of $\boldsymbol{u}$, we can have different choices depending on the physical make-up of the interfaces. For example, if the elastomer surfaces are rigidly pinned to the substrates so that the elastic displacements at the interfaces cannot undergo solenoidal motion, we can specify the rigid pinning boundary condition for $\mathbf{v}^{\perp}$, i.e.,

$$
\mathbf{v}^{\perp}(z=0)=\mathbf{v}^{\perp}(z=L)=0,
$$

whilst the corresponding BC for $\mathbf{v}^{\|}$can be found from the local incompressibility constraint. On the other hand, if we allow for some solenoidal "slippage" of the elastomer film at the interfaces, then instead of the rigid pinning BC we have additional terms (the soft "gluing" potentials, to be described in Sec. (V) in $H_{e l}$ that describe the energetic cost of slippage. In this case, the non-detachment BC still holds for $u_{z}$.
We need to calculate the fluctuation correction to the mean-field behavior described by Eq. (3.3). The fluctuation calculation is made somewhat more challenging by the presence of the local incompressibility constraint in the partition function. The corresponding strategy we adopt is to make use of a certain mode decomposition that automatically enforces the local incompressibility constraint and also shows the partition function to be a functional integral over two independent field degrees of freedom. We therefore next turn to the mode decomposition.

\section{B. Mode decomposition}

Let us write (in real space) $\mathbf{u}=(\mathbf{v}, \phi)$ where $\mathbf{v} \equiv$ $\left(u_{x}, u_{y}\right)$ and $\phi \equiv u_{z}$. Let us also define the differential operator in the $x-y$ directions: $\nabla_{\perp} \equiv\left(\partial_{x}, \partial_{y}\right)$. The uniaxially compressed elastomer is described by the Hamiltonian

$$
\begin{aligned}
H_{\mathbf{u}}= & \frac{\mu_{0}}{2} \int_{0}^{L} d z \int d^{2} \rho\left(\frac{1}{\lambda} \partial_{i} v_{\mu} \partial_{i} v_{\mu}+\lambda^{2} \partial_{i} \phi \partial_{i} \phi\right) \\
= & \frac{\mu_{0}}{2} \int_{0}^{L} d z \int d^{2} \rho\left(\frac{1}{\lambda}\left(\nabla_{\perp} \cdot \mathbf{v}^{\|}\right)^{2}+\frac{1}{\lambda}\left(\partial_{z} \mathbf{v}^{\|}\right)^{2}\right. \\
& \left.+\frac{1}{\lambda}\left(\nabla_{\perp} \times \mathbf{v}^{\perp}\right)^{2}+\frac{1}{\lambda}\left(\partial_{z} \mathbf{v}^{\perp}\right)^{2}+\lambda^{2} \partial_{i} \phi \partial_{i} \phi\right)
\end{aligned}
$$

where the Greek index $\mu=1,2$ and the Latin index $i=1,2,3$. As the co-planar substrates break translation symmetry in the $z$-direction but leave the system translationally invariant in the $x-y$ plane, the two-dimensional inverse Fourier transforms of $\mathbf{v}^{\|}$and $\mathbf{v}^{\perp}$ are given by

$$
\begin{aligned}
\mathbf{v}^{\|}(\rho, z) & =\int \frac{d^{2} \mathbf{Q}}{(2 \pi)^{2}} e^{i \rho \cdot \mathbf{Q}} \widetilde{\mathbf{v}}^{\|}(\mathbf{Q}, z), \\
\mathbf{v}^{\perp}(\rho, z) & =\int \frac{d^{2} \mathbf{Q}}{(2 \pi)^{2}} e^{i \rho \cdot \mathbf{Q}} \widetilde{\mathbf{v}}^{\perp}(\mathbf{Q}, z),
\end{aligned}
$$

and the inverse Fourier transform of $\phi$ is given by

$$
\phi(\rho, z)=\int \frac{d^{2} \mathbf{Q}}{(2 \pi)^{2}} e^{i \rho \cdot \mathbf{Q}} \widetilde{\phi}(\mathbf{Q}, z) .
$$

Here $\mathbf{Q}=\left(Q_{x}, Q_{y}\right)$ is the two-dimensional wave-vector conjugate to $\rho=(x, y)$. In two-dimensional Fourier space the properties of $\mathbf{v}^{\| l}$ and $\mathbf{v}^{\perp}$ are described by $\widehat{Q}_{\mu} \widetilde{v}_{\mu}^{\perp}=0$ and $\epsilon_{\mu \nu} \widehat{Q}_{\mu} \widetilde{v}_{\nu}^{\|}=0$ (where $\widehat{Q} \equiv \mathbf{Q} / Q, \epsilon_{12}=-\epsilon_{21}=1$ and $\left.\epsilon_{11}=\epsilon_{22}=0\right)$, which implies they can be expressed in terms of scalar modes $\chi$ and $\psi$, viz.,

$$
\widetilde{v}_{\mu}^{\|} \equiv \widehat{Q}_{\mu} \chi, \quad \widetilde{v}_{\mu}^{\perp} \equiv \epsilon_{\mu \nu} \widehat{Q}_{\nu} \psi
$$


In terms of $\chi, \psi$ and $\widetilde{\phi}$, we can rewrite $H_{\mathbf{u}}$ as

$$
\begin{aligned}
& H_{\mathbf{u}}[\chi, \psi, \phi] \\
= & \frac{\mu_{0}}{2 \lambda} \int_{0}^{L} d z \int \frac{d^{2} \mathbf{Q}}{(2 \pi)^{2}}\left(Q^{2}|\psi(\mathbf{Q}, z)|^{2}+\left|\partial_{z} \psi(\mathbf{Q}, z)\right|^{2}\right. \\
& +Q^{2}|\chi(\mathbf{Q}, z)|^{2}+\left|\partial_{z} \chi(\mathbf{Q}, z)\right|^{2} \\
& \left.+\lambda^{3}\left(Q^{2}|\widetilde{\phi}(\mathbf{Q}, z)|^{2}+\left|\partial_{z} \widetilde{\phi}(\mathbf{Q}, z)\right|^{2}\right)\right)
\end{aligned}
$$

The corresponding partition function is given by

$$
\begin{aligned}
Z_{\mathbf{u}}= & \int \mathcal{D} \chi \int \mathcal{D} \psi \int \mathcal{D} \widetilde{\phi} \prod_{\{\mathbf{Q}\}\{z\}} \prod_{\{z} \delta\left(Q \chi(\mathbf{Q}, z)-i \partial_{z} \widetilde{\phi}(\mathbf{Q}, z)\right) \\
& \times e^{-\beta H_{\mathbf{u}}[\chi, \psi, \widetilde{\phi}]}
\end{aligned}
$$

We turn next to the computation of the free energy for the two following types of boundary conditions for $\psi$ : (i) rigid pinning $\mathrm{BC}$ and (ii) "gluing" potential (to be described in Sec. VD.

\section{ELASTOMER RIGIDLY PINNED AT THE INTERFACES}

\section{A. Fluctuation modes}

First we consider the case of an elastomer rigidly pinned at the interfaces with the co-planar substrates. This means that we implement both the non-detachment $\mathrm{BC}$ (Eq. (3.7) ) for $\widetilde{\phi}$ and the rigid pinning BC (Eq. (3.8) ) for $\psi$, so these fluctuation fields are given by sinusoidal Fourier series:

$$
\begin{aligned}
& \widetilde{\phi}(\mathbf{Q}, z)=\sum_{n=1}^{\infty} \sqrt{\frac{2}{L}} \sin \left(\frac{n \pi z}{L}\right)\left(\phi_{n}^{\mathrm{re}}(\mathbf{Q})+i \phi_{n}^{\mathrm{im}}(\mathbf{Q})\right), \\
& \psi(\mathbf{Q}, z)=\sum_{n=1}^{\infty} \sqrt{\frac{2}{L}} \sin \left(\frac{n \pi z}{L}\right)\left(\psi_{n}^{\mathrm{re}}(\mathbf{Q})+i \psi_{n}^{\mathrm{im}}(\mathbf{Q})\right) .
\end{aligned}
$$

Here the superscripts "re" and "im" refer to real and imaginary parts. The independent fluctuation degrees of freedom along the $z$-direction are now replaced by the independent discrete modes labeled by $n$, where $n=1,2,3, \ldots$ To determine $\chi(\mathbf{Q}, z)$, we return to the local incompressibility constraint: $-\partial_{z}(\operatorname{Im} \widetilde{\phi}(\mathbf{Q}, z))=$ $Q \operatorname{Re} \chi(\mathbf{Q}, z)$ and $\partial_{z}(\operatorname{Re} \widetilde{\phi}(\mathbf{Q}, z))=Q \operatorname{Im} \chi(\mathbf{Q}, z)$. Used in conjunction with Eq. (4.1a), we obtain

$$
\chi(\mathbf{Q}, z)=\sum_{n=1}^{\infty} \sqrt{\frac{2}{L}}\left(\frac{n \pi}{Q L}\right) \cos \left(\frac{n \pi z}{L}\right)\left(-\phi_{n}^{\mathrm{im}}+i \phi_{n}^{\mathrm{re}}\right) .
$$

In terms of the discrete modes we can write Eq. 3.13) as

$$
\begin{aligned}
H_{\mathbf{u}}\left[\left\{\psi_{n}, \phi_{n}\right\}\right]= & \frac{\mu_{0}}{2 \lambda} \sum_{n=1}^{\infty} \int \frac{d^{2} \mathbf{Q}}{(2 \pi)^{2}}\left\{\left(Q^{2}+\left(\frac{n \pi}{L}\right)^{2}\right)\right. \\
& \times\left[\left(\psi_{n}^{\mathrm{re}}(\mathbf{Q})\right)^{2}+\left(\psi_{n}^{\mathrm{im}}(\mathbf{Q})\right)^{2}\right] \\
& +\left(\lambda^{3} Q^{2}+\left(\frac{n \pi}{L}\right)^{2}\right)\left(1+\left(\frac{n \pi}{Q L}\right)^{2}\right) \\
& \left.\times\left[\left(\phi_{n}^{\mathrm{re}}(\mathbf{Q})\right)^{2}+\left(\phi_{n}^{\mathrm{im}}(\mathbf{Q})\right)^{2}\right]\right\}
\end{aligned}
$$

As we show in Appendix B, this leads to the following fluctuation contribution to the free energy of elastic deformation:

$$
F_{\mathbf{u}}(\lambda)=\frac{k_{\mathrm{B}} T S}{2} \sum_{n=1}^{\infty} \int \frac{d^{2} \mathbf{Q}}{(2 \pi)^{2}} \ln \left(\lambda Q^{2}+\frac{1}{\lambda^{2}}\left(\frac{n \pi}{L}\right)^{2}\right)
$$

We can rewrite Eq. (4.4) as

$$
F_{\mathbf{u}}(\lambda)=\frac{k_{\mathrm{B}} T S}{4 \pi} \int_{0}^{\pi \xi^{-1}} d Q Q \sum_{n=1}^{M}\left[\ln \left(a^{2}+n^{2}\right)-2 \ln \lambda+B\right],
$$

where $a \equiv \lambda^{3 / 2} Q L / \pi, B \equiv \pi^{2} M k_{\mathrm{B}} T S / 4 L \xi^{2}$ is a term independent of $\lambda$ (and which we will thus ignore), and we have set an upper limit $M \equiv L / \xi$ on the discrete sum, as the number of fluctuating degrees of freedom in the $z$-direction is limited by the typical localization length $\xi$.

\section{B. Fluctuation free energy}

Before we turn to evaluate the free energy of the confined elastomer film, we make a few general remarks about the anticipated features of such a free energy. In a confined elastomer film, thermal fluctuations of the elastic matrix introduce qualitative changes to the free energy and the value of $\lambda$ that minimizes the free energy. In an isotropic bulk elastomer, such elastic fluctuations cause a spontaneous change in the volume of the undeformed system (relative to the state of the undeformed elastomer in mean-field theory [12]), but the value of $\lambda$ that characterizes the undeformed state remains unchanged at unity [11]. The latter is expected on grounds of symmetry as it is equally energetically costly for elastic fluctuations to occur in every direction in space, the elastomer being equally macroscopically large (and homogeneous) in every direction. In this respect, the reference (i.e., undeformed) space can be regarded as isotropic and translationally invariant in every direction. On the other hand, the reference space of a confined elastomer film is isotropic only in the transverse directions, and translation symmetry is broken in the direction normal to the film's surface. As we have seen, applying the Dirichlettype non-detachment $\mathrm{BC}$ on the spectral decomposition of the elastic fluctuations leads to a discrete spectrum of modes in the normal direction, whereas there is a quasicontinuous spectrum of modes in the transverse directions. Each mode carries a thermal energy, and there are 


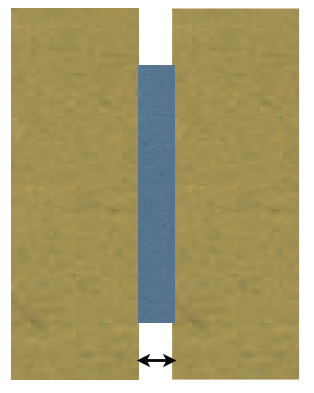

(i)

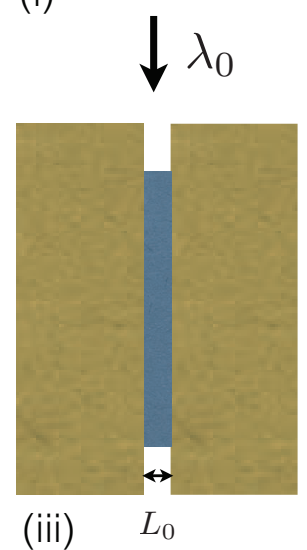

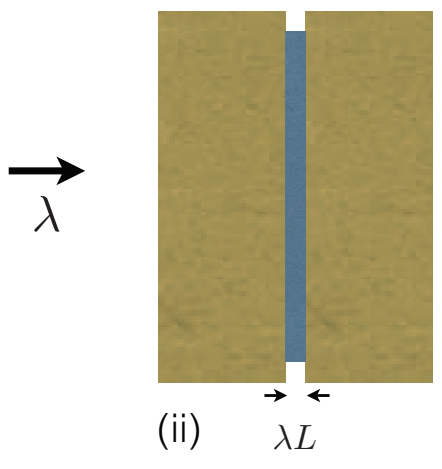

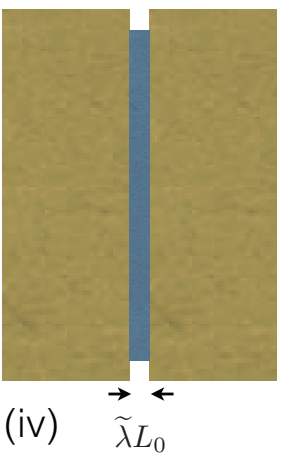

FIG. 1: Comparison of different ground states and relative deformations. The state in (i) corresponds to the undeformed or reference state in mean-field theory (where thermal fluctuations of the elastic matrix are absent). We call this the mean-field ground state, and is the ground state considered by classical rubber elasticity theory. In (ii), the elastomer undergoes an external deformation with a deformation $\lambda$ measured relative to the mean-field ground state. If we allow for the effect of thermal fluctuations of the elastic matrix, the elastomer in state (i) will undergo a spontaneous relaxation to state (iii), with a deformation $\lambda_{0}$ measured relative to the mean-field ground state. We call state (iii) the fluctuation corrected or true ground state. In (iv) the elastomer undergoes an external deformation $\tilde{\lambda}$ relative to the true ground state, and such deformation corresponds to what is measured in experiment.

many more modes in the transverse directions than in the normal direction. The spectral anisotropy will thus be reflected in the energetic anisotropy of the fluctuationcorrected free energy, which means for example that we expect that the free energy minimum should occur at a value of $\lambda$ different from unity, $\lambda$ being the strain measured relative to the isotropic undeformed state (or ground state) in mean-field theory. Hence, the fluctuations generate internal "pre-stress" that causes the system to undergo a spontaneous shear relaxation, while the physically measurable strain is defined with reference to the state that has already spontaneously relaxed.

To evaluate the free energy, we first decompose the discrete sum in Eq. (4.5) as follows:

$$
\begin{aligned}
\sum_{n=1}^{M} \ln \left(n^{2}+a^{2}\right) & =\sum_{n=1}^{M}(\ln (n+i a)+\ln (n-i a)) \\
& =\ln \left((1+i a)_{M}+(1-i a)_{M}\right) \\
& =\ln \left[\frac{\Gamma(M+1-i a) \Gamma(M+1+i a)}{\Gamma(1+i a) \Gamma(1-i a)}\right]
\end{aligned}
$$

In the above, the Pochhammer symbol $(x)_{n}$ denotes $\Gamma(x+n) / \Gamma(x)$. We make use of the result (see e.g., Ref. [21])

$$
\Gamma(1+i a) \Gamma(1-i a)=\frac{\pi a}{\sinh (\pi a)}
$$

and in the limit that $z \gg 1$, use Stirling's approximation to $\Gamma(z)$ [21]

$$
\Gamma(z) \approx(z / e)^{z} \sqrt{2 \pi / z}
$$

We thus have

$$
\begin{aligned}
& \Gamma(M+1-i a) \Gamma(M+1+i a) \\
\approx & 2 \pi e^{(M+1-i a) \ln (M+1-i a)-(M+1-i a)} \\
& \times e^{(M+1+i a) \ln (M+1+i a)-(M+1+i a)} \\
& \times e^{-\frac{1}{2} \ln (M+1-i a)-\frac{1}{2} \ln (M+1+i a)} \\
= & 2 \pi e^{\left(M+\frac{1}{2}\right) \ln \left((M+1)^{2}+a^{2}\right)} \\
& \times e^{-2 a \tan ^{-1}\left(\frac{a}{M+1}\right)-2(M+1)},
\end{aligned}
$$

where we have used the identity $\tan ^{-1} z=(i / 2) \ln ((1-$ $i z) /(1+i z))$ [22]. By writing $L / \xi=M \approx M+1 / 2$, we have

$$
\begin{gathered}
\ln (\Gamma(M+1-i a) \Gamma(M+1+i a)) \\
\approx \ln 2 \pi+\frac{L}{\xi} \ln \left[\left(\frac{L}{\xi}\right)^{2}+\lambda^{3}\left(\frac{Q L}{\pi}\right)^{2}\right] \\
-\frac{2 \lambda^{3 / 2} Q L}{\pi} \tan ^{-1}\left(\frac{\lambda^{3 / 2} Q \xi}{\pi}\right)-\frac{2 L}{\xi} .
\end{gathered}
$$

Using (4.5), (4.6) and (4.9), the discrete sum can be put in the form

$$
\begin{aligned}
& \sum_{n=1}^{M} \ln \left(a^{2}+n^{2}\right) \\
\approx & M \ln \left[1+\left(\frac{a}{M}\right)^{2}\right]+2 a \cot ^{-1}\left(\frac{a}{M}\right) \\
& -\ln \pi a+\ln \left(1-e^{-2 \pi a}\right)+C,
\end{aligned}
$$

where we used the identity $\tan ^{-1}(z)=\pi / 2-\cot ^{-1}(z)$ and approximated $M+1$ and $M+\frac{1}{2}$ by $M$, which is valid for large $M$. The term $C \equiv \ln \pi+2 M \ln M-2 M$ is independent of $\lambda$, and we can thus disregard the corresponding term in the free energy. In Fig. 2, we compare the left-hand side (LHS) and right-hand side (RHS) of Eq. (4.10), with the LHS and RHS evaluated relative to their values at $\lambda=1$, for $M=5$ and $M=10$, and $\lambda=0.3$ 


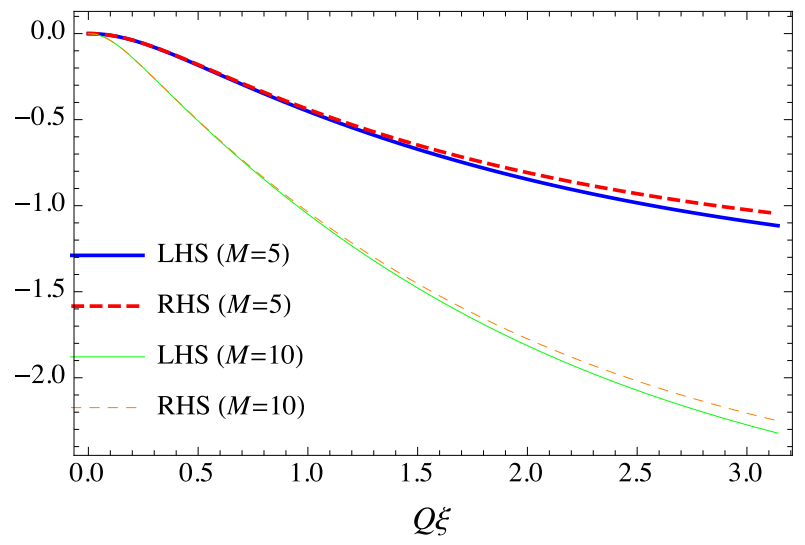

(i)

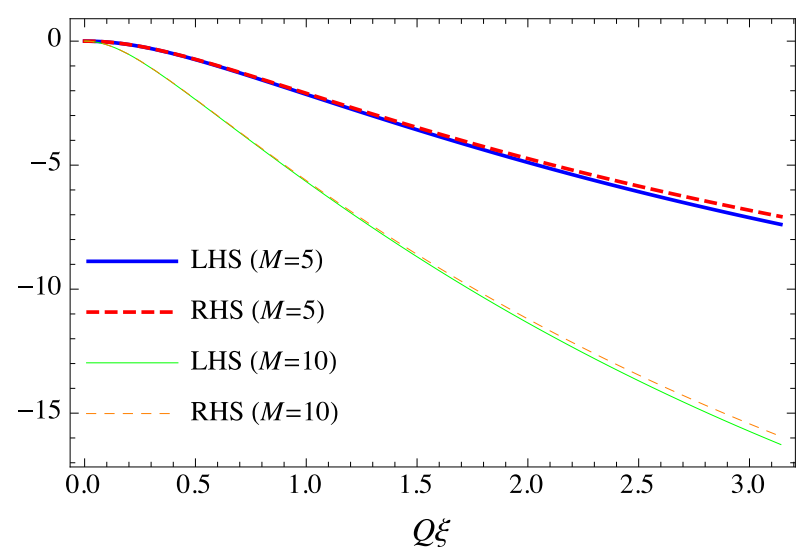

(ii)

FIG. 2: Comparison of the left-hand side (LHS) and righthand side (RHS) of Eq. (4.10), for (i) $\lambda=0.9$ and (ii) $\lambda=0.3$, with the LHS and RHS evaluated relative to their values at $\lambda=1$. For each case we plot the behavior for $M=5$ and $M=10$.

and $\lambda=0.9$. We see that the agreement between the exact LHS and its approximation in the RHS improves for larger values of $M$ and smaller values of $\lambda$. Furthermore, for each value of $\lambda$ and $M$, the agreement is better for the lower half-range of values of $Q$, with the error becoming more noticeable only for $Q$ close to the upper bound $\pi \xi^{-1}$ (the upper bound being there because continuum elasticity is not defined on lengthscales smaller than the typical localization length).

Writing $M=L / \xi$, we have

$$
\begin{aligned}
& F_{\mathbf{u}}(\lambda) \\
\approx & \frac{k_{\mathrm{B}} T S}{4 \pi} \int_{0}^{\pi \xi^{-1}} d Q Q\left\{\ln \left(1-e^{-2 \lambda^{3 / 2} Q L}\right)-\ln \lambda^{3 / 2}\right. \\
& -\frac{2 L}{\xi} \ln \lambda+\frac{L}{\xi} \ln \left[1+\lambda^{3}\left(\frac{Q \xi}{\pi}\right)^{2}\right] \\
& \left.+\frac{2 \lambda^{3 / 2} Q L}{\pi} \cot ^{-1}\left(\frac{\lambda^{3 / 2} Q \xi}{\pi}\right)\right\}
\end{aligned}
$$

where we have neglected terms independent of $\lambda$. The first term describes the interaction between the confining surfaces, the second term can be interpreted as a surface tension term, and the remaining terms are the bulk terms. The first term resembles, but is not identical to, the standard form of a Casimir free energy (see, e.g., Ref. [15]), the differences being the finite upper cut-off and the presence of $\lambda$ in the exponent. Strictly speaking, even in studies of the Casimir effect, a finite upper cutoff (which corresponds to the smallest length scale in the problem) should be imposed 23]; however, the difference between the integral with a finite upper cut-off and one with an infinite upper bound is an irrelevant constant in the usual Casimir problems, which do not involve timepersistent elastic stresses in the intervening medium, and can thus be ignored. In our present problem, we cannot replace the integral by one with an infinite upper bound, because the difference depends on $\lambda$ and thus changes as the elastomer is deformed.

We can rewrite the integral over the first term as the sum

$$
\begin{aligned}
& \int_{0}^{\pi \xi^{-1}} d Q Q \ln \left(1-e^{-2 \lambda^{3 / 2} Q L}\right) \\
= & -\frac{\zeta_{\mathrm{R}}(3)}{4 \lambda^{3} L^{2}}-g(\lambda, L), \\
& g(\lambda, L) \equiv \int_{\pi \xi^{-1}}^{\infty} d Q Q \ln \left(1-e^{-2 \lambda^{3 / 2} Q L}\right) .
\end{aligned}
$$

In the above, $\zeta_{\mathrm{R}}(s) \equiv \sum_{n=1}^{\infty} 1 / n^{s}$ is the Riemann zeta function, and $\zeta_{\mathrm{R}}(3) \approx 1.202[21]$. We thus obtain for the fluctuation free energy:

$$
\begin{aligned}
F_{\mathbf{u}}(\lambda) \approx & -\frac{k_{\mathrm{B}} T S \zeta_{\mathrm{R}}(3)}{16 \pi \lambda^{3} L^{2}}-\frac{k_{\mathrm{B}} T S}{4 \pi} g(\lambda, L) \\
& -\frac{3 \pi k_{\mathrm{B}} T S}{16 \xi^{2}} \ln \lambda+\frac{\pi k_{\mathrm{B}} T V}{24 \xi^{3}} f_{\text {bulk }} .
\end{aligned}
$$

In the above, we denote the volume by $V \equiv S L$, and

$$
\begin{aligned}
f_{\text {bulk }} \equiv & 4 \lambda^{3 / 2} \cot ^{-1}\left(\lambda^{3 / 2}\right)-6 \ln \lambda-1 \\
& +\left(3+\frac{1}{\lambda^{3}}\right) \ln \left(1+\lambda^{3}\right) .
\end{aligned}
$$

The first three terms in Eq. 4.13) scale as $S$, whereas the rest are bulk terms that scale as $V$ [24].

\section{Reference state}

The full free energy is given by the sum of the meanfield contribution (Eq. (3.3)) and the fluctuation correction:

$$
F_{\text {full }}=F_{0}+F_{\mathbf{u}} .
$$

$F_{0}$ has a minimum at $\lambda=1$ but the minimum of $F_{\text {full }}$ occurs at $\lambda=\lambda_{0} \neq 1$. We can understand this by noting 


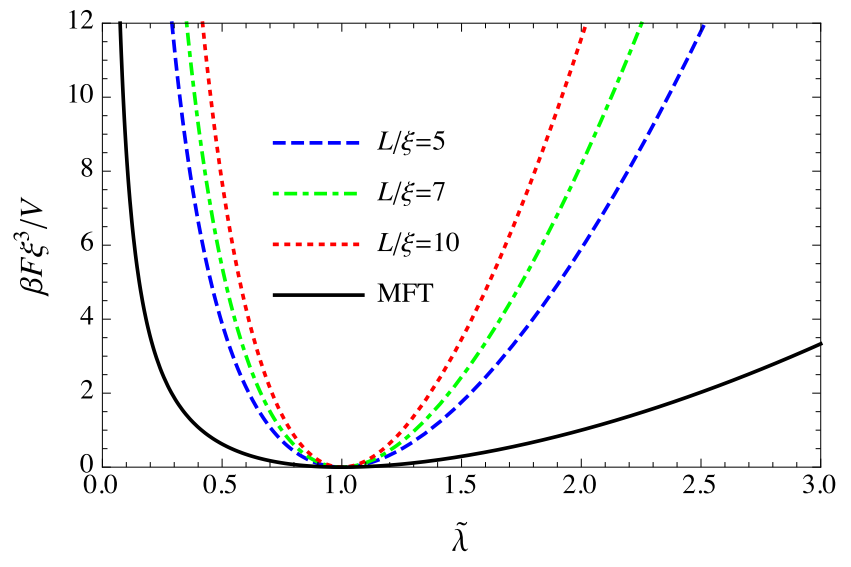

FIG. 3: Comparison of the free energy densities of elastic deformation as a function of deformation $\widetilde{\lambda}$ measured relative to the true ground state (i.e., undeformed state of the elastomer after elastic fluctuations have been allowed to relax), for $\mu_{0}=k_{\mathrm{B}} T / \xi^{3}$ and (i) $L=5 \xi$ (blue, dashed), (ii) $L=7 \xi$ (green, dot-dashed), and (iii) $L=10 \xi$ (red, dotted). Comparison is made with the mean-field elastic energy in Eq. (3.3) (black).

that $\lambda=1$ is the ground state of the mean-field theory, viz.,

$$
\left.\frac{\partial F_{0}}{\partial \lambda}\right|_{\lambda=1}=0
$$

which corresponds to a thickness $L$ measured in a state where the positions of the cross-links (i.e., the ambient elastic matrix) do not undergo thermal fluctuations. On the other hand, in a state where the elastic matrix does undergo thermal fluctuations, the fluctuations will cause the system to relax to a new equilibrium thickness $L_{0}$ distinct from $L$ (see Fig. 1). The corresponding value of $\lambda$ (where $\lambda$ is a deformation relative to the mean-field ground state) is $\lambda_{0} \equiv L_{0} / L$, and $\lambda_{0}$ is determined from the stationarity condition:

$$
\left.\frac{\partial F_{\text {full }}}{\partial \lambda}\right|_{\lambda=\lambda_{0}}=0
$$

The value of $\lambda_{0}$ can be determined numerically. For example, for $\mu_{0}=k_{\mathrm{B}} T / \xi^{3}, \lambda_{0} \approx 1.0466$ for $L=5 \xi$, $\lambda_{0} \approx 1.03717$ for $L=7 \xi$ and $\lambda_{0} \approx 1.03008$ for $L=10 \xi$. We call the state that satisfies Eq. (4.17), the true ground state. If we measure a subsequent, isothermal, externally applied deformation relative to the true ground state, the corresponding strain $\tilde{\lambda}=L^{\prime} / L_{0}$ (where $L^{\prime}$ is the thickness of the deformed elastomer) is related to $\lambda$ via

$$
\lambda=\widetilde{\lambda} \lambda_{0}
$$

\section{Deformation free energy}

The full deformation free energy is given by

$$
F(\widetilde{\lambda})=F_{\text {full }}\left(\widetilde{\lambda} \lambda_{0}\right)-F_{\text {full }}\left(\lambda_{0}\right)
$$

This quantity vanishes for zero external deformation relative to the true ground state $[F(\widetilde{\lambda}=1)=0]$. Using Eqs. (3.3) and (4.11), we find the deformation free energy for a system with rigid pinning $\mathrm{BC}$ :

$$
\begin{aligned}
& \left(\beta \xi^{3} / V\right) F(\widetilde{\lambda}) \\
= & \frac{\beta \xi^{3} \mu_{0}}{2}\left[\left(\widetilde{\lambda} \lambda_{0}\right)^{2}+\frac{2}{\widetilde{\lambda} \lambda_{0}}-\lambda_{0}^{2}-\frac{2}{\lambda_{0}}\right] \\
+ & \frac{\xi^{3}}{4 \pi L} \int_{0}^{\pi \xi^{-1}} d Q Q \ln \left[\frac{1-e^{-2\left(\widetilde{\lambda} \lambda_{0}\right)^{3 / 2} Q L}}{1-e^{-2 \lambda_{0}^{3 / 2} Q L}}\right] \\
+ & \frac{\pi}{6}\left[\left(\widetilde{\lambda} \lambda_{0}\right)^{3 / 2} \cot ^{-1}\left(\left(\widetilde{\lambda} \lambda_{0}\right)^{3 / 2}\right)-\lambda_{0}^{3 / 2} \cot ^{-1}\left(\lambda_{0}^{3 / 2}\right)\right] \\
+ & \frac{\pi}{24}\left[3+\frac{1}{\left(\widetilde{\lambda} \lambda_{0}\right)^{3}}\right] \ln \left(1+\left(\widetilde{\lambda} \lambda_{0}\right)^{3}\right) \\
& -\frac{\pi}{24}\left[3+\frac{1}{\lambda_{0}^{3}}\right] \ln \left(1+\lambda_{0}^{3}\right)-\frac{\pi}{4} \ln \widetilde{\lambda}-\frac{3 \pi \xi}{16 L} \ln \widetilde{\lambda}
\end{aligned}
$$

In Fig. 3. we show the behavior of the free energy density as a function of $\widetilde{\lambda}$ for three different thicknesses of the elastomer film, $L=5 \xi, 7 \xi$ and $10 \xi$ [25]. We see that elastic fluctuations introduce a significant deviation from the qualitative behavior predicted by mean-field theory; in particular, fluctuations raise the overall free energy of the system relative to what mean-field theory predicts, and the increase is more significant for larger film thicknesses. For example, the full free energy can be seventy times larger than the mean-field value if an elastomer of undeformed thickness $5 \xi$ is compressed to $70 \%$ of its original thickness.

\section{ELASTOMER "GLUED" AT THE INTERFACES}

\section{A. Hamiltonian}

Next, we consider the case of an elastomer that is "glued" at the interfaces, so that the solenoidal elastic fluctuations can undergo some slippage there. This is analogous, for example, to hydrodynamic slippage of a fluid in a nanopore due to a hydrophobic mismatch between the fluid and the surface of the nanopore [26]. In practice the glue can be an adhesive such as polysaccharide adhesive viscous exopolysaccharide (PAVE) isolated from the marine bacterium Alteromonas colwelliana [27]. Instead of the rigid pinning BC we have a "soft" gluing potential at the confining surfaces [28 32], described by 
two extra terms in $H_{u}$ :

$$
\begin{aligned}
H_{\mathbf{u}}= & \frac{\mu_{0}}{2} \int_{0}^{L} d z \int d^{2} \rho\left(\frac{1}{\lambda}\left(\nabla_{\perp} \cdot \mathbf{v}^{\|}\right)^{2}+\frac{1}{\lambda}\left(\partial_{z} \mathbf{v}^{\|}\right)^{2}\right. \\
& \left.+\frac{1}{\lambda}\left(\nabla_{\perp} \times \mathbf{v}^{\perp}\right)^{2}+\frac{1}{\lambda}\left(\partial_{z} \mathbf{v}^{\perp}\right)^{2}+\lambda^{2} \partial_{i} \phi \partial_{i} \phi\right) \\
& +\frac{K_{t}}{2 w_{0}^{2}} \int d^{2} \rho\left(\left|\mathbf{v}^{\perp}(z=0)\right|^{2}+\left|\mathbf{v}^{\perp}(z=L)\right|^{2}\right)
\end{aligned}
$$

The terms within the integral over $z$ are the same as those in Eq. (3.9), whereas the last two terms describe the energetic cost of elastomer slippage at the interface. $K_{t}$ quantifies the slippage energy cost (or adhesion strength) per unit area on each of the two interfaces, and $w_{0}$ has the meaning of a "slippage length". We have only written down soft BC terms for $\mathbf{v}^{\perp}$ as the other component $\mathbf{v}^{\|}$is completely determined by $\phi$ via the local incompressibility constraint. In terms of $\widetilde{\phi}, \chi$ and $\psi$ (defined by Eqs. (3.11) and (3.12)), we now have

$$
\begin{aligned}
& H_{\mathbf{u}}[\chi, \psi, \widetilde{\phi}] \\
= & \frac{\mu_{0}}{2 \lambda} \int_{0}^{L} d z \int \frac{d^{2} \mathbf{Q}}{(2 \pi)^{2}}\left(Q^{2}|\psi(\mathbf{Q}, z)|^{2}+\left|\partial_{z} \psi(\mathbf{Q}, z)\right|^{2}\right. \\
& +Q^{2}|\chi(\mathbf{Q}, z)|^{2}+\left|\partial_{z} \chi(\mathbf{Q}, z)\right|^{2} \\
& \left.+\lambda^{3}\left(Q^{2}|\widetilde{\phi}(\mathbf{Q}, z)|^{2}+\left|\partial_{z} \widetilde{\phi}(\mathbf{Q}, z)\right|^{2}\right)\right) \\
& +\frac{K_{t}}{2 w_{0}^{2}} \int \frac{d^{2} \mathbf{Q}}{(2 \pi)^{2}}\left(|\psi(\mathbf{Q}, z=0)|^{2}+|\psi(\mathbf{Q}, z=L)|^{2}\right) .
\end{aligned}
$$

The form of the above expression is consistent with the recovery of Eq. (4.1) in the limit that $K_{t} \rightarrow \infty$ (which corresponds to the regime of rigid pinning).

The non-detachment BC (Eq. (3.7)) taken together with the local incompressibility condition means that $\phi$ and $\chi$ are still given by Eqs. (4.1a) and (4.2), respectively. The Hamiltonian is then given by

$$
H_{\mathbf{u}}\left[\left\{\phi_{n}^{\mathrm{re}}, \phi_{n}^{\mathrm{im}}\right\}, \psi\right]=H_{1}+H_{\psi},
$$

where

$$
\begin{aligned}
H_{1} \equiv & \frac{\mu_{0}}{2 \lambda} \sum_{n=1}^{\infty} \int \frac{d^{2} \mathbf{Q}}{(2 \pi)^{2}}\left(\lambda^{3} Q^{2}+\left(\frac{n \pi}{L}\right)^{2}\right)\left(1+\left(\frac{n \pi}{Q L}\right)^{2}\right) \\
& \times\left[\left(\phi_{n}^{\mathrm{re}}(\mathbf{Q})\right)^{2}+\left(\phi_{n}^{\mathrm{im}}(\mathbf{Q})\right)^{2}\right]
\end{aligned}
$$

and

$$
\begin{aligned}
H_{\psi} \equiv & \frac{\mu_{0}}{2 \lambda} \int_{0}^{L} d z \int \frac{d^{2} \mathbf{Q}}{(2 \pi)^{2}}\left(Q^{2}|\psi(\mathbf{Q}, z)|^{2}+\left|\partial_{z} \psi(\mathbf{Q}, z)\right|^{2}\right) \\
& +\frac{K_{t}}{2 w_{0}^{2}} \int \frac{d^{2} \mathbf{Q}}{(2 \pi)^{2}}\left(|\psi(\mathbf{Q}, z=0)|^{2}+|\psi(\mathbf{Q}, z=L)|^{2}\right) .
\end{aligned}
$$

Next, we turn to evaluate the partition function.

\section{B. Partition function}

The fluctuation contribution to the partition function can be expressed as

$$
Z_{\mathbf{u}}=Z_{1} Z_{\psi}
$$

where

$$
\begin{aligned}
& Z_{1} \equiv \prod_{\substack{\{\mathbf{Q}>0\} \\
\left\{n \in Z^{+}\right\}}} \int d \phi_{n}^{\mathrm{re}}(\mathbf{Q}) \int d \phi_{n}^{\mathrm{im}}(\mathbf{Q}) e^{-\beta H_{1}} \\
& Z_{\psi} \equiv \prod_{\substack{\{\mathbf{Q}>0\} \\
\{z \in[0, L]\}}} \int d \psi^{\mathrm{re}}(\mathbf{Q}, z) \int d \psi^{\mathrm{im}}(\mathbf{Q}, z) e^{-\beta H_{\psi}}(
\end{aligned}
$$

In the above, $Z^{+}$refers to the set of all positive integers and $\{\mathbf{Q}>\mathbf{0}\}$ refers to the set of all positive wave-vectors ("positivity" being defined with reference to a straight line that divides the two-dimensional lattice of points $\left(Q_{x}, Q_{y}\right)$ into two halves; e.g., if we denote the normal vector to such a line by $\mathbf{n}$, then a wave-vector is positive if it satisfies $\mathbf{Q} \cdot \mathbf{n}>0$ ). To evaluate $Z_{\psi}$ we note that it has the form of a (Euclidean) Feynman path integral for a harmonic oscillator where $z$ is a time-like coordinate, and accordingly we apply the Fourier series method of Feynman and Hibbs [36]. Let us define the Hamiltonian density $\widehat{H}_{\psi}(\mathbf{Q})$ in $\mathbf{Q}$-space:

$$
H_{\psi} \equiv \int \frac{d^{2} \mathbf{Q}}{(2 \pi)^{2}} \widehat{H}_{\psi}(\mathbf{Q}),
$$

and write

$$
\psi(\mathbf{Q}, z)=\psi_{\mathrm{cl}}(\mathbf{Q}, z)+q(\mathbf{Q}, z)
$$

where $\psi_{\mathrm{cl}}(\mathbf{Q}, z)$ is a solution to the saddle-point equation

$$
\partial_{z}^{2} \psi_{\mathrm{cl}}=Q^{2} \psi_{\mathrm{cl}}
$$

and have the values $\psi_{\mathrm{cl}}(\mathbf{Q}, z=0)=X_{\mathbf{Q}}$ and $\psi_{\mathrm{cl}}(\mathbf{Q}, z=$ $L)=Y_{\mathbf{Q}}$ at the boundary interfaces, whilst

$$
q(\mathbf{Q}, z)=\sum_{n} \psi_{n}(\mathbf{Q}) \sin \left(\frac{n \pi z}{L}\right)
$$

and $q(\mathbf{Q}, z)$ satisfy Dirichlet boundary conditions. For fluctuations of given wave-vector $\mathbf{Q}$ and specified boundary values $\psi(\mathbf{Q}, z=0)=X_{\mathbf{Q}}$ and $\psi(\mathbf{Q}, z=L)=Y_{\mathbf{Q}}$, we have made a decomposition into (i) a "classical trajectory" $\psi_{\mathrm{cl}}(\mathbf{Q}, z)$ that extremizes the Boltzmann weight factor $e^{-\beta \widehat{H}_{\psi}(\mathbf{Q})}$, and (ii) deviations $q(\mathbf{Q}, z)$ about this trajectory, with the same "end-points" (i.e., zero fluctuation amplitude at the boundaries). [Note that the case of $\psi(\mathbf{Q}, z)$ subject to Dirichlet BC (considered in Sec. IV] is a special case where $\psi_{\mathrm{cl}}(\mathbf{Q}, z) \equiv 0$.] Tracing over all fluctuations in $Z_{\psi}$ is then equivalent to tracing over all deviations $q$ for the same classical trajectory for given boundary values, and then tracing over all possible 


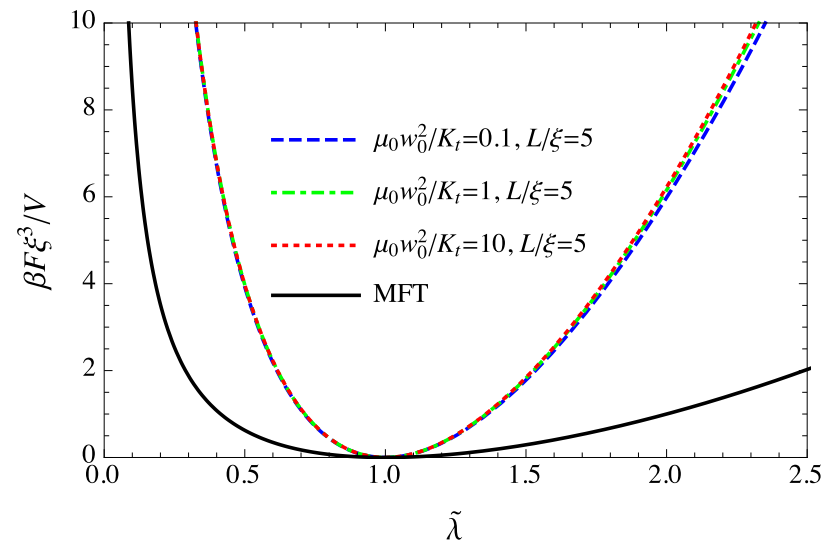

FIG. 4: Free energy behavior of a system with a soft gluing potential, $L=5 \xi$ and $\mu_{0}=k_{\mathrm{B}} T / \xi^{3}$, for $\mu_{0} w_{0}^{2} / K_{t}=0.1$ (blue dashed), $\mu_{0} w_{0}^{2} / K_{t}=1$ (green dot-dashed), and $\mu_{0} w_{0}^{2} / K_{t}=$ 10 (red dotted). For comparison we display the behavior predicted by classical rubber elasticity (black), with $L=\xi$ and $\mu_{0}=k_{\mathrm{B}} T$.

boundary values. The above-mentioned decomposition also ensures that the Hamiltonian separates into two decoupled contributions:

$$
\begin{aligned}
\widehat{H}_{\psi}(\mathbf{Q})= & \frac{\mu_{0}}{2 \lambda} \int_{0}^{L} d z\left(Q^{2}\left|\psi_{\mathrm{cl}}(\mathbf{Q}, z)\right|^{2}+\left|\partial_{z} \psi_{\mathrm{cl}}(\mathbf{Q}, z)\right|^{2}\right) \\
& +\frac{K_{t}}{2 w_{0}^{2}}\left(\left|\psi_{\mathrm{cl}}(\mathbf{Q}, z=0)\right|^{2}+\left|\psi_{\mathrm{cl}}(\mathbf{Q}, z=L)\right|^{2}\right) \\
& +\frac{\mu_{0}}{2 \lambda} \int_{0}^{L} d z\left(Q^{2}|q(\mathbf{Q}, z)|^{2}+\left|\partial_{z} q(\mathbf{Q}, z)\right|^{2}\right) .
\end{aligned}
$$

Accordingly, we find after implementing boundary conditions that

$$
\psi_{\mathrm{cl}}(\mathbf{Q}, z)=\frac{Y_{\mathbf{Q}}-X_{\mathbf{Q}} \cosh Q L}{\sinh Q L} \sinh Q z+X_{\mathbf{Q}} \cosh Q z .
$$

Substituting Eqs. (5.12) and (5.14) into Eq. (5.13) and summing over wave-vectors, we obtain

$$
\begin{aligned}
& H_{\psi} \equiv \sum_{n} \int \frac{d^{2} \mathbf{Q}}{(2 \pi)^{2}} \frac{\mu_{0} L}{4 \lambda}\left(Q^{2}+\frac{n^{2} \pi^{2}}{L^{2}}\right) \\
& \times\left[\left(\psi_{n}^{\mathrm{re}}(\mathbf{Q})\right)^{2}+\left(\psi_{n}^{\mathrm{im}}(\mathbf{Q})\right)^{2}\right] \\
&+\frac{1}{2} \int \frac{d^{2} \mathbf{Q}}{(2 \pi)^{2}}\left[\left(\frac{\mu_{0} Q}{\lambda} \operatorname{coth} Q L+\frac{K_{t}}{w_{0}^{2}}\right)\left(\left|X_{\mathbf{Q}}\right|^{2}+\left|Y_{\mathbf{Q}}\right|^{2}\right)\right. \\
&\left.\quad-\frac{2 \mu_{0} Q}{\lambda}(\operatorname{cosech} Q L)\left(X_{\mathbf{Q}}^{\mathrm{re}} Y_{\mathbf{Q}}^{\mathrm{re}}+X_{\mathbf{Q}}^{\mathrm{im}} Y_{\mathbf{Q}}^{\mathrm{im}}\right)\right] \cdot(5.15)
\end{aligned}
$$

The evaluation of $Z_{1}$ and $Z_{\psi}$ in Eq. (5.6) involves a straightforward Gaussian functional integration, and is carried out in Appendix C] The result for $Z_{\mathbf{u}}$ is Eq. (C4). Next, we turn to evaluate the deformation free energy.

\section{Free energy}

The fluctuation free energy is evaluated in Appendix $\mathrm{C}$, and the result is given by

$$
F_{\mathbf{u}}=-k_{\mathrm{B}} T \ln Z=F_{1}+F_{2}+\text { const, }
$$

where the "const" refers to contributions that are independent of $\lambda$, and

$$
\begin{aligned}
F_{1} \equiv & \frac{k_{\mathrm{B}} T S}{4 \pi} \int_{0}^{\pi \xi^{-1}} d Q Q \ln \left(1-e^{-2 \lambda^{3 / 2} Q L}\right) \\
& -\frac{3 \pi k_{\mathrm{B}} T S}{16 \xi^{2}} \ln \lambda+\frac{\pi k_{\mathrm{B}} T V}{24 \xi^{3}} f_{\mathrm{bulk}}, \\
F_{2} \equiv & \frac{k_{\mathrm{B}} T S}{4 \pi} \int_{0}^{\pi \xi^{-1}} d Q Q \ln \left[(1+\alpha(\lambda) \operatorname{coth} Q L)^{2}\right. \\
& \left.-(\alpha(\lambda) \operatorname{cosech} Q L)^{2}\right] \\
= & \frac{k_{\mathrm{B}} T S}{4 \pi} \int_{0}^{\pi \xi^{-1}} d Q Q\left\{2 \ln (1+\alpha(\lambda))-\ln \left(1-e^{-2 Q L}\right)\right. \\
& \left.+\ln \left[1-\left(\frac{1-\alpha(\lambda)}{1+\alpha(\lambda)}\right)^{2} e^{-2 Q L}\right]\right\},
\end{aligned}
$$

where $\alpha(\lambda) \equiv \mu_{0} w_{0}^{2} Q /\left(\lambda K_{t}\right)$. The term $f_{\text {bulk }}$ is given by Eq. (4.14). The contribution $F_{1}$ is the same as the fluctuation free energy for an elastomer with rigid pinning BC (cf. Eqs. (4.11) and (4.13) of Sec. IV), whilst $F_{2}$ is the extra contribution that arises from the finite strength of the gluing potential. The first term of $F_{2}$ can be interpreted as a surface tension term, whilst the second and third terms describe the interaction between the confining surfaces. As we expect, the last term in the formula for $F_{2}$ has a similar form to the thermal Casimir free energy for a slab with soft boundary conditions and no region exterior to the slab 28 32].

As in Eq. (4.19) of Sec. IV] the full deformation energy is given by

$$
F(\widetilde{\lambda})=F_{\text {full }}\left(\widetilde{\lambda} \lambda_{0}\right)-F_{\text {full }}\left(\lambda_{0}\right),
$$

where $F_{\text {full }} \equiv F_{0}+F_{1}+F_{2}$. For $L=5 \xi$, we find, by numerically solving for $\lambda_{0}$ in the stationarity condition Eq. (4.17), viz., $\left.\left(\partial F_{\text {full }}(\lambda) / \partial \lambda\right)\right|_{\lambda=\lambda_{0}}=0$, that $\lambda_{0} \approx 1.05414$ for $\mu_{0} w_{0}^{2} / K_{t}=0.1, \lambda_{0} \approx 1.07503$ for $\mu_{0} w_{0}^{2} / K_{t}=1$, and $\lambda_{0} \approx 1.08807$ for $\mu_{0} w_{0}^{2} / K_{t}=10$. As we do not know the actual value of the adhesion strength $K_{t}$, we have tried a range of values from small to large [33, 34]. The corresponding deformation free energy behavior is displayed in Fig. 4. The deformation free energy is larger for smaller gluing strengths $K_{t}$, because more fluctuation modes can be excited, and each mode contributes thermal energy to the overall free energy.

\section{ELASTIC PSEUDO-CASIMIR STRESS}

We now turn to explore the fluctuation-induced, or pseudo-Casimir, stresses that lead the system to spontaneously relax to the true ground state, in particular 


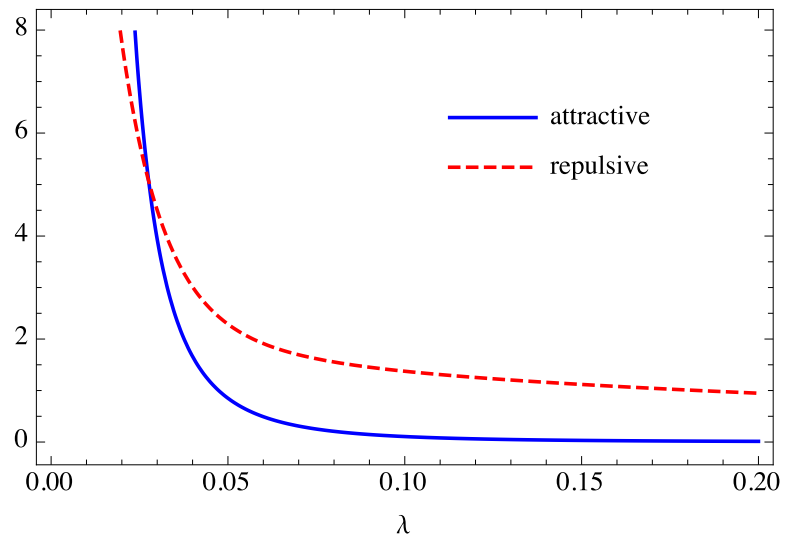

FIG. 5: Comparison (in dimensionless units) of the magnitudes of the attractive, pseudo-Casimir term, i.e., $\zeta_{\mathrm{R}}(3) \xi^{2} / 16 \pi \lambda^{3} L^{2}$ (blue solid), and the repulsive and finitesize contributions to the surface free energy part of Eq. (4.13), i.e., $-g(\lambda, L) \xi^{2} / 4 \pi-3 \pi \ln \lambda / 16$ (red dashed), for the case $L / \xi=15$. For $\lambda=0.07$ (corresponding to a measured thickness of $L^{\prime}=1.05 \xi$, which is larger than the typical localization length), the magnitude of the former contribution is $18.3 \%$ of the latter contribution.

comparing the attractive component of such stresses with the thermal Casimir stresses of non-elastomeric systems. To consider fluctuation stresses we consider deformations defined relative to the mean-field ground state (i.e., before the system has spontaneously relaxed). As is typical in studies of the Casimir effect, we will focus on the surface free energy contribution [35]. We shall look at the effects of the rigid pinning $\mathrm{BC}$ and the soft gluing potential. First we consider the case of rigid pinning BC, and we refer to the discussion in Sec. IV] In Eq. (4.13), the first term is attractive and reminiscent of a Casimir effect:

$$
F_{\mathrm{c}} \equiv-\frac{k_{\mathrm{B}} T S \zeta_{\mathrm{R}}(3)}{16 \pi \lambda^{3} L^{2}}=-\frac{k_{\mathrm{B}} T S L \zeta_{\mathrm{R}}(3)}{16 \pi\left(L^{\prime}\right)^{3}},
$$

where $L^{\prime}=\lambda L$ is the thickness of the deformed elastomer. The pseudo-Casimir contribution $F_{\mathrm{c}}$ is thus attractive, and decays as the inverse cube of the current separation between the substrates. Besides this attractive contribution to the surface free energy, Eq. (4.13) also contains a surface term which is proportional to $-\ln \lambda$, and thus repulsive for compressions, as well as a finite-size correction (proportional to $-g(\lambda, L)$ ) to the pseudo-Casimir term. These latter two contributions to the surface free energy compete with the attractive pseudo-Casimir contribution, as we see from Fig. 5. Our problem is thus distinct from the pseudo-Casimir physics of confined nonelastomeric systems such as a nematic liquid crystal confined between two flat plates with strong homeotropic anchoring at the surface of each plate. There the director fluctuation free energy decays inversely as the square of the separation between the plates, and does not involve any additional repulsive terms originating from internal stresses of the intervening medium [15].

We determine the pseudo-Casimir stress $\sigma_{\mathrm{c}}$ from the formula

$$
\sigma_{\mathrm{c}}=-\frac{\lambda}{S} \frac{\partial F_{\mathrm{c}}(\lambda)}{L \partial \lambda}=-\frac{3 k_{\mathrm{B}} T \zeta_{\mathrm{R}}(3)}{16 \pi \lambda^{3} L^{3}}=-\frac{3 k_{\mathrm{B}} T \zeta_{\mathrm{R}}(3)}{16 \pi\left(L^{\prime}\right)^{3}},
$$

which is attractive and decays as $\left(L^{\prime}\right)^{-3}$. The right-hand side of the first equality contains a prefactor $\lambda$ to account for the change in the cross-sectional area after deformation. This prefactor is necessary to define the true stress (as opposed to nominal stress, which decays as $\left.\left(L^{\prime}\right)^{-4}\right)$. The distinction between true and nominal stress only arises because we are dealing with an incompressible elastomer [6], while in the studies of the Casimir effect in non-elastomeric systems [13 17], the Casimir stress computed corresponds to the nominal stress. Although the pseudo-Casimir stress in a confined elastomer film decays with the same power law as that in non-elastomeric systems (e.g., confined nematic and electromagnetic fluctuations), the mechanisms giving rise to the same power law are qualitatively distinct.

Next, we consider the correction to $F_{c}$ and $\sigma_{c}$ that come from a soft gluing potential (cf. Sec. V). To enable a formal comparison with the more studied case of the pseudo-Casimir effect emerging in confined nematic liquid crystals [15], consider the last term of Eq. (5.18) with the upper bound in the integral set to infinity; let us call this $\delta F_{c}$ :

$$
\delta F_{c}=\frac{k_{\mathrm{B}} T S}{4 \pi L^{2}} \int_{0}^{\infty} d u u \ln \left[1-\left(\frac{1-x u}{1+x u}\right)^{2} e^{-2 u}\right]
$$

where $u \equiv Q L$ and $x \equiv \mu_{0} w_{0}^{2} /\left(\lambda K_{t} L\right)$. The problem is analytically tractable [37] for sufficiently large $K_{t}$, where $x$ is small, and we can expand $\delta F_{c}$ in powers of $x$, obtaining:

$$
\begin{aligned}
\delta F_{c} \approx & -\frac{k_{\mathrm{B}} T S \zeta_{\mathrm{R}}(3)}{16 \pi L^{2}}+\frac{k_{\mathrm{B}} T S \zeta_{\mathrm{R}}(3) \mu_{0} w_{0}^{2}}{4 \pi \lambda K_{t} L^{3}} \\
& -\frac{3 k_{\mathrm{B}} T S \zeta_{\mathrm{R}}(3)\left(\mu_{0} w_{0}^{2}\right)^{2}}{4 \pi \lambda^{2} K_{t}^{2} L^{4}}
\end{aligned}
$$

The first term can be disregarded as it is independent of $\lambda$. The corresponding true stress is

$$
\begin{aligned}
\delta \sigma_{c} & =\frac{k_{\mathrm{B}} T \zeta_{\mathrm{R}}(3) \mu_{0} w_{0}^{2}}{4 \pi \lambda K_{t} L^{4}}-\frac{3 k_{\mathrm{B}} T \zeta_{\mathrm{R}}(3)\left(\mu_{0} w_{0}^{2}\right)^{2}}{2 \pi \lambda^{2} K_{t}^{2} L^{5}} \\
& =\frac{k_{\mathrm{B}} T \zeta_{\mathrm{R}}(3) \mu_{0} w_{0}^{2}}{4 \pi K_{t} L^{3} L^{\prime}}-\frac{3 k_{\mathrm{B}} T \zeta_{\mathrm{R}}(3)\left(\mu_{0} w_{0}^{2}\right)^{2}}{2 \pi K_{t}^{2} L^{3}\left(L^{\prime}\right)^{2}}(6.5)
\end{aligned}
$$

The leading term has a positive sign, indicating that the soft gluing potential leads to a less attractive pseudoCasimir stress, and decays as $\left(L^{\prime}\right)^{-1}$. The pseudoCasimir stress for a system with a soft gluing potential is obtained by adding $\delta \sigma_{c}$ to $\sigma_{c}$. Again, the contribution of the pseudo-Casimir stress correction is offset by the finite size correction as well as the first term of Eq. (5.18). 


\section{SUMMARY AND CONCLUSION}

In this study we have examined the important contribution of thermal fluctuations of the elastic displacement to the elasticity of thin confined elastomer films of an axisymmetric shape. We have found that there can be significant departures (both quantitative and qualitative) from the prediction of classical rubber elasticity theory when elastic fluctuations are included. Furthermore, we have also addressed the impact of different types of boundary conditions on a particular (solenoidal) mode of elastic fluctuation on the elastic deformation free energy, focussing on the effect of (i) a "rigid pinning" boundary condition and (ii) a pair of "gluing" boundary potentials (which can be regarded as the elastic analogue of hydrodynamic slippage). We found that the deformation free energy is lower in case (i) than in case (ii). In addition, we have also explored the formal similarities of the attractive component of the elastic fluctuation-induced (pseudo-Casimir) stress with the thermal Casimir stress. The attractive component for the rigid pinning $\mathrm{BC}$, as well as the leading order term corresponding to the gluing potential problem, has the same $\left(L^{\prime}\right)^{-3}$ decay, where $L^{\prime}$ is the inter-surface separation. On the other hand, there are also corrections to the attractive part of the fluctuation stress for the gluing potential problem (which stem from the finiteness of the gluing strength), and the leading correction term decays as $\left(L^{\prime}\right)^{-1}$.

Our investigation into the thermal elastic fluctuation effects between bounding surfaces in the context of confined elastomers now opens up the venue of analyzing the effective fluctuation-induced interactions between rigid inclusions in the elastomer network. Similar fluctuation mediated interactions have been introduced between e.g. protein inclusions in a background of thermal fluctuations of the lipid membrane, that exist as long as the rigidity of the inclusion differs from that of the ambient membrane [38]. Another possible venue is to investigate the analogue of the critical Casimir effect (i.e., the Casimir effect in a system characterized by an order parameter, for example a thin superfluid film, where the effect is generated by long-range fluctuations of the order parameter when the system is near-critical, such that the fluctuations approach "masslessness" [39, 40]) in elastomeric systems, for example cross-linked polymer blends under confinement. Our approach can be extended to study the effects of disorder introduced by random chemical cross-linking (disorder effects have indeed received a lot of attention recently in other examples of fluctuation-induced forces; see Refs. [38, 41 50] and references therein), and also to investigate the behavior of nematic elastomers [9, 51 53. in confined environments.

\section{ACKNOWLEDGMENT}

BSL thanks Institute for Research in Fundamental Sciences (IPM), Tehran, for a memorable stay in May 2015, where the present work was conceived. He also thanks L. Athanasopoulou for constructive discussions. BSL and $\mathrm{RP}$ would like to acknowledge the financial support of the Agency for research and development of Slovenia under Grants No. N1-0019 and P1-0055. AN acknowledges partial support from the Royal Society, the Royal Academy of Engineering, and the British Academy (UK).

\section{Appendix A: Derivation of the fluctuation Hamiltonian, Eq. (3.5)}

Here we fill in the steps leading from Eq. (3.1) to (3.5). Using Eq. (2.1), we can express Eq. (3.1) as

$$
\begin{aligned}
Z=\int & \mathcal{D} \mathbf{u} \delta(\nabla \cdot \mathbf{u}) \exp \left\{-\frac{\beta \mu_{0}}{2} \int_{0}^{L} d z \int d^{2} x_{\perp}\left[\Lambda_{i a} \Lambda_{i a}\right.\right. \\
& \left.\left.+2 \Lambda_{i a} \Lambda_{i b} \frac{\partial u_{b}}{\partial x_{a}}+\Lambda_{i b} \Lambda_{i c} \frac{\partial u_{b}}{\partial x_{a}} \frac{\partial u_{c}}{\partial x_{a}}\right]\right\}
\end{aligned}
$$

Let us define a matrix $g_{a b} \equiv \Lambda_{i a} \Lambda_{i b}$ and a vector $u_{a}^{\prime} \equiv$ $g_{a b} u_{b}$. In terms of the new variable and using $\delta(\nabla \cdot \mathbf{u})=$ $\operatorname{det} \underline{\underline{g}} \delta\left(\partial_{a} g_{a b} u_{b}\right)$, we can express $Z$ as

$$
\begin{aligned}
Z= & \int \mathcal{D} \mathbf{u}^{\prime} \mathcal{J} \delta\left(\nabla \cdot \mathbf{u}^{\prime}\right) \operatorname{det} \underline{\underline{g}} \\
& \times \exp \left\{-\frac{\beta \mu_{0}}{2} \int_{0}^{L} d z \int d^{2} x_{\perp}\left[\Lambda_{i a} \Lambda_{i a}+2 \Lambda_{i a} \Lambda_{i b} g_{b c}^{-1} \frac{\partial u_{c}^{\prime}}{\partial x_{a}}\right.\right. \\
& \left.\left.+\Lambda_{i b} \Lambda_{i c} g_{b b^{\prime}}^{-1} g_{c c^{\prime}}^{-1} \frac{\partial u_{b^{\prime}}}{\partial x_{a}} \frac{\partial u_{c^{\prime}}}{\partial x_{a}}\right]\right\}
\end{aligned}
$$

Here $\mathcal{J} \equiv\left\|\delta \mathbf{u} / \delta \mathbf{u}^{\prime}\right\|$ is the functional Jacobian for the transformation of field variables $\mathbf{u}$ to $\mathbf{u}^{\prime}$. As $\Lambda_{i a} \Lambda_{i b} g_{b c}^{-1}=$ $g_{a b} g_{b c}^{-1}=\delta_{a c}$, the second term in the exponent is proportional to $\nabla \cdot \mathbf{u}^{\prime}$. The Dirac delta-function $\delta\left(\nabla \cdot \mathbf{u}^{\prime}\right)$ is only non-zero for configurations for which $\nabla \cdot \mathbf{u}^{\prime}=0$, implying that we can set the second term of the exponent to zero. Next we make a change of variables from $\mathbf{u}^{\prime}$ back to $\mathbf{u}$. Equation (A2) then becomes

$$
Z=\int \mathcal{D} \mathbf{u} \delta(\nabla \cdot \mathbf{u}) e^{-\beta\left(\frac{\mu_{0} V}{2} \operatorname{Tr}\left(\underline{\underline{\Lambda}}^{\mathrm{T}} \cdot \underline{\underline{\Lambda}}\right)+H_{\mathbf{u}}\right)}
$$

where $H_{\mathbf{u}}$ is given by Eq. (3.5).

\section{Appendix B: Derivation of the fluctuation free energy, Eq. (4.4)}

The fluctuation Hamiltonian after the constraint of local incompressibility has been applied, is given by 
Eq. (4.3), viz.,

$$
\begin{aligned}
H_{\mathbf{u}}\left[\left\{\psi_{n}, \phi_{n}\right\}\right]= & \frac{\mu_{0}}{2 \lambda} \sum_{n=1}^{\infty} \int \frac{d^{2} \mathbf{Q}}{(2 \pi)^{2}}\left\{\left(Q^{2}+\left(\frac{n \pi}{L}\right)^{2}\right)\right. \\
& \times\left[\left(\psi_{n}^{\mathrm{re}}(\mathbf{Q})\right)^{2}+\left(\psi_{n}^{\mathrm{im}}(\mathbf{Q})\right)^{2}\right] \\
+ & \left(\lambda^{3} Q^{2}+\left(\frac{n \pi}{L}\right)^{2}\right)\left(1+\left(\frac{n \pi}{Q L}\right)^{2}\right) \\
& \left.\times\left[\left(\phi_{n}^{\mathrm{re}}(\mathbf{Q})\right)^{2}+\left(\phi_{n}^{\mathrm{im}}(\mathbf{Q})\right)^{2}\right]\right\}
\end{aligned}
$$

The partition function $Z_{\mathbf{u}}$ with the incompressibility constraint and Dirichlet BC taken into account can consequently be expressed in Fourier space as

$$
\begin{aligned}
Z_{\mathbf{u}}= & \prod_{\substack{\{\mathbf{Q}>0\} \\
\left\{n \in Z^{+}\right\}}} \int d \phi_{n}^{\mathrm{re}}(\mathbf{Q}) d \phi_{n}^{\mathrm{im}}(\mathbf{Q}) \int d \psi_{n}^{\mathrm{re}}(\mathbf{Q}) d \psi_{n}^{\mathrm{im}}(\mathbf{Q}) \\
& \times e^{-\beta H_{\mathbf{u}}\left[\left\{\phi_{n}^{\mathrm{re}}, \phi_{n}^{\mathrm{im}}, \psi_{n}^{\mathrm{re}}, \psi_{n}^{\mathrm{im}}\right\}\right]}
\end{aligned}
$$

The Hamiltonian is Gaussian in the fluctuations, and thus the functional integrals over the fluctuating fields can be straightforwardly performed, yielding

$$
\begin{aligned}
Z_{\mathbf{u}}= & \prod_{\substack{\{\mathbf{Q}>\mathbf{>}\} \\
\{n \in Z\}}}\left[\frac{2 \pi k_{\mathrm{B}} T \lambda S}{\mu_{0}\left(Q^{2}+\left(\frac{n \pi}{L}\right)^{2}\right)}\right] \\
& \times\left[\frac{2 \pi k_{\mathrm{B}} T \lambda S}{\mu_{0}\left(\lambda^{3} Q^{2}+\left(\frac{n \pi}{L}\right)^{2}\right)\left(1+\left(\frac{n \pi}{Q L}\right)^{2}\right)}\right] \\
= & e^{A-\beta F_{\mathbf{u}}(\lambda)}
\end{aligned}
$$

where $Z^{+}$refers to the set of positive integers, $\{\mathbf{Q}>\mathbf{0}\}$ refers to the set of all positive wave-vectors ("positivity" being defined with reference to a straight line that divides the two-dimensional lattice of points $\left(Q_{x}, Q_{y}\right)$ into two halves; e.g., if we denote the normal vector to such a line by $\mathbf{n}$, then a wave-vector is positive if it satisfies $\mathbf{Q} \cdot \mathbf{n}>0), A$ is a constant defined by

$A \equiv \sum_{n=1}^{\infty} \sum_{\{\mathbf{Q}>\mathbf{0}\}}\left\{\ln \left[\frac{4 \pi^{2} S^{2}\left(k_{\mathrm{B}} T\right)^{2} Q^{2}}{\mu_{0}^{2}}\right]-2 \ln \left[Q^{2}+\left(\frac{n \pi}{L}\right)^{2}\right]\right\}$

and $F_{\mathbf{u}}$ is given by

$$
F_{\mathbf{u}} \equiv k_{\mathrm{B}} T \sum_{n=1}^{\infty} \sum_{\{\mathbf{Q}>\mathbf{0}\}} \ln \left[\lambda Q^{2}+\frac{1}{\lambda^{2}}\left(\frac{n \pi}{L}\right)^{2}\right]
$$

In the above, $S$ is the cross-sectional area of the surface of the elastomer film that is co-planar with the confining substrates, in the state prior to external deformation. The functional integral runs over all independent fluctuating field degrees of freedom. As the real and imaginary components of the (complex) fluctuating fields $\phi_{\mathbf{Q}}$ and $\psi_{\mathbf{Q}}$ obey the relations $\phi_{-\mathbf{Q}}^{\mathrm{re}}=\phi_{\mathbf{Q}}^{\mathrm{re}}, \phi_{-\mathbf{Q}}^{\mathrm{im}}=-\phi_{\mathbf{Q}}^{\mathrm{im}}$ (and similar ones for $\psi_{\mathbf{Q}}$, these relations being required by the reality of the fluctuating fields in real space), the modes with positive and negative wave-vectors $\mathbf{Q}$ are not really independent of each other, and thus the functional integral product runs only over the positive wavenumber contributions. By making the continuum limit $\sum_{\{\mathbf{Q}\}}=S \int d^{2} \mathbf{Q} /(2 \pi)^{2}$ where the wave-vector sum now runs over all wave-vectors, we have

$$
F_{\mathbf{u}}=\frac{k_{\mathrm{B}} T S}{2} \sum_{n=1}^{\infty} \int \frac{d^{2} \mathbf{Q}}{(2 \pi)^{2}} \ln \left[\lambda Q^{2}+\frac{1}{\lambda^{2}}\left(\frac{n \pi}{L}\right)^{2}\right],
$$

which is Eq. (4.4).

\section{Appendix C: Derivation of the fluctuation free energy for a "glued" elastomer, Eq. (5.16)}

In this Section, we provide the calculational steps to derive the fluctuation free energy in Eq. (5.16) for an elastomer "glued" to the substrates. The partition function $Z_{\mathbf{u}}$ corresponding to Eq. (5.2) is given by Eq. (5.6). Similar to the step from Eq. (B2) to (B3), the evaluation of Eq. (5.7) involves functionally integrating over the sets of fluctuating fields $\left\{\phi_{n}^{\mathrm{re}}\right\}$ and $\left\{\phi_{n}^{\mathrm{im}}\right\}$ which are Gaussian in form (as we can see from Eq. (5.4)). The functional integration thus yields

$$
Z_{1}=\prod_{\substack{\{\mathbf{Q}>0\} \\\left\{n \in Z^{+}\right\}}}\left[\frac{2 \pi k_{\mathrm{B}} T S}{\mu_{0}\left(\lambda^{2} Q^{2}+\frac{1}{\lambda}\left(\frac{n \pi}{L}\right)^{2}\right)\left(1+\left(\frac{n \pi}{Q L}\right)^{2}\right)}\right]
$$

To evaluate $Z_{\psi}$ (cf. Eq. (5.8)), we have to functionally integrate over six sets of fluctuating fields, viz., $\left\{\psi_{n}^{\text {re }}\right\}$, $\left\{\psi_{n}^{\mathrm{im}}\right\},\left\{X_{\mathbf{Q}}^{\mathrm{re}}\right\},\left\{X_{\mathbf{Q}}^{\mathrm{im}}\right\},\left\{Y_{\mathbf{Q}}^{\mathrm{re}}\right\}$, and $\left\{Y_{\mathbf{Q}}^{\mathrm{im}}\right\}$. Again, as we see from Eq. (5.15), these fields are Gaussian in form. Making use of the formula

$$
\int_{-\infty}^{\infty} d X \int_{-\infty}^{\infty} d Y e^{-\frac{a}{2}\left(X^{2}+Y^{2}\right)+b X Y}=\frac{2 \pi}{\sqrt{a^{2}-b^{2}}},
$$

with the identification $a=\frac{\mu_{0} Q}{\lambda} \operatorname{coth} Q L+\frac{K_{t}}{w_{0}^{2}}$ and $b=$ $\frac{\mu_{0} Q}{\lambda}(\operatorname{cosech} Q L)$, and $H_{\psi}$ from Eq. (5.15), we have

$$
\begin{aligned}
Z_{\psi}= & \prod_{\substack{\{\mathbf{Q}>0\} \\
\left\{n \in Z^{+}\right\}}} d \psi_{n}^{\mathrm{re}}(\mathbf{Q}) d \psi_{n}^{\mathrm{im}}(\mathbf{Q}) \int d X_{\mathbf{Q}}^{\mathrm{re}} d X_{\mathbf{Q}}^{\mathrm{im}} \int d Y_{\mathbf{Q}}^{\mathrm{re}} d Y_{\mathbf{Q}}^{\mathrm{im}} \\
= & \prod_{\substack{\{\mathbf{Q}>\mathbf{0}\} \\
\left\{n \in Z^{+}\right\}}} \frac{4 \pi \lambda k_{\mathrm{u}}\left[\left\{\psi_{n}^{\mathrm{re}}, \psi_{n}^{\mathrm{im}}, X_{\mathbf{Q}}^{\mathrm{re}}, X_{\mathbf{Q}}^{\mathrm{im}}, Y_{\mathbf{Q}}^{\mathrm{re}}, Y_{\mathbf{Q}}^{\mathrm{im}}\right\}\right]}{\left.L \mu^{2}+\left(\frac{n \pi}{L}\right)^{2}\right)} \\
& \times \frac{4 \pi^{2}\left(k_{\mathrm{B}} T\right)^{2} S}{\left(\frac{\mu_{0} Q}{\lambda} \operatorname{coth} Q L+\frac{K_{t}}{w_{0}^{2}}\right)^{2}-\left(\frac{\mu_{0} Q}{\lambda} \operatorname{cosech} Q L\right)^{2}}, \quad(\mathrm{C} 3)
\end{aligned}
$$

where the first factor in the right-hand side of the second equality comes from a functional integration over $\psi_{n}^{\text {re }}(\mathbf{Q})$ and $\psi_{n}^{\mathrm{im}}(\mathbf{Q})$, and the second factor comes from a functional integration over $X_{\mathbf{Q}}^{\mathrm{re}}, X_{\mathbf{Q}}^{\mathrm{im}}, Y_{\mathbf{Q}}^{\mathrm{re}}$, and $Y_{\mathbf{Q}}^{\mathrm{im}}$. 
Using Eqs. (C1) and (C3), we find that the partition function $Z_{\mathbf{u}}$ in Eq. (5.6) is given by

$$
\begin{aligned}
= & \prod_{\substack{\{\mathbf{Q}>\mathbf{0}\} \\
\left\{n \in Z^{+}\right\}}} \frac{32 \pi^{4}\left(k_{\mathrm{B}} T\right)^{4} w_{0}^{4} S^{3}}{L \mu_{0}^{2} K_{t}^{2} Q^{2}\left(1+\left(\frac{n \pi}{Q L}\right)^{2}\right)^{2}\left[\lambda Q^{2}+\frac{1}{\lambda^{2}}\left(\frac{n \pi}{L}\right)^{2}\right]} \\
& \times \frac{1}{(1+\alpha(\lambda) \operatorname{coth} Q L)^{2}-(\alpha(\lambda) \operatorname{cosech} Q L)^{2}}
\end{aligned}
$$

where $\alpha(\lambda) \equiv \mu_{0} w_{0}^{2} Q /\left(\lambda K_{t}\right)$. The fluctuation free energy is given by $F_{\mathbf{u}}=-k_{\mathrm{B}} T \ln Z_{\mathbf{u}}$, i.e.,

$$
\begin{aligned}
& F_{\mathbf{u}} \\
& =D+k_{\mathrm{B}} T \sum_{\{\mathbf{Q}>\mathbf{0}\}}\left\{\sum_{n=1}^{\infty} \ln \left[\lambda Q^{2}+\frac{1}{\lambda^{2}}\left(\frac{n \pi}{L}\right)^{2}\right]\right. \\
& \left.\quad+\ln \left[(1+\alpha(\lambda) \operatorname{coth} Q L)^{2}-(\alpha(\lambda) \operatorname{cosech} Q L)^{2}\right]\right\},
\end{aligned}
$$

where $D$ is independent of $\lambda$, given by

$$
\begin{aligned}
D \equiv- & k_{\mathrm{B}} T \sum_{\{\mathbf{Q}>\mathbf{0}\}}\left\{\ln \frac{32 \pi^{4}\left(k_{\mathrm{B}} T\right)^{4} w_{0}^{4} S^{3}}{L \mu_{0}^{2} K_{t}^{2}}\right. \\
& \left.-\sum_{n=1}^{\infty} \ln Q^{2}\left[1+\left(\frac{n \pi}{Q L}\right)^{2}\right]^{2}\right\}
\end{aligned}
$$

The second term of Eq. C5 is identical to the term in Eq. (B5), which we can write as $F_{1}$, where

$$
F_{1} \equiv \frac{k_{\mathrm{B}} T S}{2} \sum_{n=1}^{\infty} \int \frac{d^{2} \mathbf{Q}}{(2 \pi)^{2}} \ln \left(\lambda Q^{2}+\frac{1}{\lambda^{2}}\left(\frac{n \pi}{L}\right)^{2}\right)
$$

Making use of Eqs. (4.12) and (4.13) allows us to rewrite $F_{1}$ as Eq. (5.17).

We can rewrite the third term of Eq. (C5) as $F_{2}$, where

$$
\begin{gathered}
F_{2} \equiv k_{\mathrm{B}} T \sum_{\{\mathbf{Q}>\mathbf{0}\}} \ln \left[(1+\alpha(\lambda) \operatorname{coth} Q L)^{2}\right. \\
\left.-(\alpha(\lambda) \operatorname{cosech} Q L)^{2}\right] \\
=\frac{k_{\mathrm{B}} T S}{2} \int \frac{d^{2} \mathbf{Q}}{(2 \pi)^{2}} \ln \left[(1+\alpha(\lambda) \operatorname{coth} Q L)^{2}\right. \\
\left.-(\alpha(\lambda) \operatorname{cosech} Q L)^{2}\right] .
\end{gathered}
$$

In the second step we have made the continuum limit, and we thus arrive at Eq. (5.18). Summing up the contributions $D, F_{1}$ and $F_{2}$ gives us the fluctuation free energy, Eq. (5.16).
[1] M. Zhai and G. B. McKenna, "Elastic modulus and surface tension of a polyurethane rubber in nanothick films" Polymer 55, 2725 (2014).

[2] G. Harsanyi, Polymer Films in Sensor Applications (CRC Press, 1995).

[3] L. Zhai, Chem. Soc. Rev. 42, 7148 (2013).

[4] R. H. Pritchard, Y.Y.S. Huang and E. M. Terentjev, "Mechanics of biological networks: from the cell cytoskeleton to connective tissue" Soft Matter 10, 1864 (2014).

[5] C. P. Broedersz, F. C. MacKintosh, "Modeling semiflexible polymer networks". Rev. Mod. Phys. 86, 995 (2014).

[6] L. R. G. Treloar, The Physics of Rubber Elasticity (Clarendon Press, Oxford, 1975).

[7] H. M. James and E. Guth, "Theory of the Elastic Properties of Rubber", J. Chem. Phys. 11, 455 (1943)

[8] M. Rubinstein and S. Panyukov, "Elasticity of polymer networks", Macromolecules 35, 6670-6686 (2002).

[9] M. Warner and E. M. Terentjev, Liquid Crystal Elastomers (Oxford University Press, Oxford, 2003).

[10] P. M. Goldbart, H. E. Castillo and A. Zippelius, "Randomly Crosslinked Macromolecular Systems: Vulcanisation Transition to and Properties of the Amorphous Solid State", Adv. Phys. 45, 393 (1996)

[11] X. Xing, P. M. Goldbart and L. Radzihovsky, "Thermal Fluctuations and Rubber Elasticity", Phys. Rev. Lett. 98, 075502 (2007)

[12] X. Mao, P. M. Goldbart, X. Xing and A. Zippelius, "Soft random solids and their heterogeneous elasticity", Phys. Rev. E 80, 031140 (2009)
[13] M. Bordag, G. L. Klimchitskaya, U. Mohideen, and V. M. Mostepanenko, Advances in the Casimir Effect (Oxford University Press, Oxford, 2009).

[14] V. M. Mostepanenko and N. N. Trunov, The Casimir Effect and its Applications (Oxford University Press, Oxford, 1997).

[15] A. Ajdari, B. Duplantier, D. Hone, L. Peliti, and J. Prost, "Pseudo-Casimir effect in liquid crystals", J. de Physique II 2, 487-501 (1992)

[16] P. Ziherl, R. Podgornik, S. Žumer, "Casimir force in liquid crystals close to the nematic-isotropic phase transition", Chem. Phys. Letts. 295, 99-104 (1998)

[17] J. Dobnikar and R. Podgornik, "Pseudo-Casimir force in confined nematic polymers", Europhys. Lett. 53, 735-741 (2001)

[18] M. Kardar and R. Golestanian, "The 'friction' of vacuum, and other fluctuation-induced forces", Rev. Mod. Phys. 71, 1233 (1999)

[19] R. Courant and D. Hilbert, Methods of Mathematical Physics, Volume II (Wiley-Interscience, 1962)

[20] F. Closa, F. Ziebert, and E. Raphael, "Interplay of internal stresses, electric stresses, and surface diffusion in polymer films", Phys. Rev. E 83, 051603 (2011).

[21] E. T. Whittaker and G. N. Watson, A Course of Modern Analysis (4th edition, Cambridge University Press, Cambridge, UK, 1927), Sect. 12.

[22] This identity can be found for example in the article "Inverse Tangent" from Wolfram MathWorld, available at http://mathworld.wolfram.com/InverseTangent.html

[23] V. M. Mostepanenko and N. N. Trunov, "The Casimir 
effect and its applications", Sov. Phys. Usp. 31, 965-987 (1989); Usp. Fiz. Nauk. 156, 385-426 (1988).

[24] We observe that we do not recover the elastic fluctuation free energy of Ref. [11] in the bulk limit. This is because we consider an elastomer which is of axisymmetric shape in its undeformed state, and thus remains anisotropic in the bulk limit, whereas Ref. 11] considered an elastomer which is spherical when undeformed, and thus isotropic.

[25] These values for $L$ are consistent with the thicknesses of rubber films studied, e.g. in Ref. [1], if we take the typical localization length to be of nanometer order (see, e.g., Ref. [10]). The thicknesses of ultrathin polyurethane rubber can range between $22 \mathrm{~nm}$ and $220 \mathrm{~nm}$.

[26] L. Bocquet and E. Charlaix, "Nanofluidics, from bulk to interfaces", Chem. Soc. Rev. 39, 1073 (2010).

[27] A. M. Smith and J. A. Callow, Biological Adhesives (Springer-Verlag, Heidelberg, Germany, 2006).

[28] D. S. Dean, "Thermal Casimir effect with soft boundary conditions", Phys. Rev. E 79, 011108 (2009).

[29] F. M. Schmidt and H. W. Diehl, "Crossover from attractive to repulsive Casimir forces and vice versa", Phys. Rev. Lett. 101, 100601 (2008).

[30] L. C. de Albuquerque and R. M. Cavalcanti, "Casimir effect for the scalar field under Robin boundary conditions: a functional integral approach", J. Phys. A: Math. Gen. 37, 7039 (2004).

[31] Z. Bajnok, L. Palla, and G. Takacs, "Casimir force between planes as a boundary finite size effect", Phys. Rev. D 73065001 (2006).

[32] A. Romeo and A. A. Saharian, "Casimir effect for scalar fields under Robin boundary conditions on plates", J. Phys. A: Math. Gen. 351297 (2002).

[33] F. K. Pour Haddadan, A. Naji, A. Kh. Seifi, R. Podgornik, "Pseudo-Casimir interactions across nematic films with disordered anchoring axis", J. Phys.: Condens. Matter 26, 075103 (2014).

[34] F. K. Pour Haddadan, A. Naji, N. Shirzadiani, R. Podgornik, "Fluctuation-induced interactions in nematics with disordered anchoring energy", J. Phys.: Condens. Matter 26, 505101 (2014).

[35] M. Krech, The Casimir Effect in Critical Systems (World Scientific Publishing Co., Singapore, 1994).

[36] R. P. Feynman and A. R. Hibbs, Quantum Mechanics and Path Integrals (McGraw-Hill Companies, Inc., New York, 1965)

[37] R. Podgornik, "Solvent structure effects in dipole correlation forces", Chem. Phys. Lett. 144, 503-508 (1988).

[38] H. Li and M. Kardar, "Fluctuation-induced forces between manifolds immersed in correlated fluids" Phys. Rev. A 46, 6490-6500 (1992); "Fluctuation-induced forces between rough surfaces" Phys. Rev. Lett. 67, 32753278 (1991).

[39] C. Hertlein, L. Helden, A. Gambassi, S. Dietrich, and C. Bechinger, "Direct measurement of critical Casimir forces" Nature 451, 172-175 (2008)

[40] A. Gambassi, A. Maciolek, C. Hertlein, U. Nellen, L. Helden, C. Bechinger, and S. Dietrich, "Critical Casimir effect in classical binary liquid mixtures", Phys. Rev. E 80, 061143 (2009)

[41] D.S. Dean, J. Dobnikar, A. Naji, R. Podgornik (Eds.), Electrostatics of Soft and Disordered Matter (Pan Stanford Publishing, Singapore, 2014).

[42] R. Podgornik, A. Naji, Europhys. Lett. 74, 712 (2006).

[43] A. Naji, D.S. Dean, J. Sarabadani, R. Horgan, R. Podgornik, "Fluctuation-Induced Interaction between Randomly Charged Dielectrics", Phys. Rev. Lett. 104, 060601 (2010).

[44] J. Sarabadani, A. Naji, D.S. Dean, R.R. Horgan and R. Podgornik, "Nonmonotonic Fluctuation-Induced Interactions between Dielectric Slabs carrying Charge Disorder", J. Chem. Phys. 133, 174702 (2010).

[45] D.S. Dean, A. Naji and R. Podgornik, "ample-toSample Fluctuations of Electrostatic Forces Generated by Quenched Charge Disorder", Phys. Rev. E 83, 011102 (2011).

[46] A. Naji, J. Sarabadani, D.S. Dean and R. Podgornik, "Sample-to-Sample Torque Fluctuations in a System of Coaxial Randomly Charged Surfaces", Eur. Phys. J. E 35, 24 (2012).

[47] V. Rezvani, J. Sarabadani, A. Naji and R. Podgornik, "Electromagnetic Fluctuation-Induced Interactions in Randomly Charged Slabs", J. Chem. Phys. 137, 114704 (2012).

[48] F. Karimi Pour Haddadan, A. Naji, A. Kh. Seifi, R. Podgornik, "Pseudo-Casimir Interactions across Nematic Films with Disordered Anchoring Axis", J. Phys.: Condens. Matter 26, 075103 (2014); Corrigendum: J. Phys.: Condens. Matter 26, 179501 (2014).

[49] F. Karimi Pour Haddadan, A. Naji, N. Shirzadiani, R. Podgornik, "Fluctuation-Induced Interactions in Nematics with Disordered Anchoring Energy", J. Phys.: Condens. Matter 26, 505101 (2014).

[50] B.-S. Lu, A. Naji, R. Podgornik, "Molecular Recognition by Van der Waals Interaction between Polymers with Sequence-Specific Polarizabilities", J. Chem. Phys. 142, 214904 (2015).

[51] G. Skačej and C. Zannoni, "Molecular simulations shed light on supersoft elasticity in polydomain liquid crystal elastomers", Macromolecules 47, 8824 (2014)

[52] B.-S. Lu, F. Ye, X. Xing and P. M. Goldbart, "Phenomenological Theory of Isotropic-Genesis Nematic Elastomers", Phys. Rev. Lett. 108, 257803 (2012)

[53] B.-S. Lu, F. Ye, X. Xing and P. M. Goldbart, "Statistical physics of isotropic-genesis nematic elastomers: I. Structure and correlations at high temperatures", Int. J. Mod. Phys. B 27, 1330012 (2013) 